\title{
Distinct Roles of Different Neural Cell Adhesion Molecule (NCAM) Isoforms in Synaptic Maturation Revealed by Analysis of NCAM $180 \mathrm{kDa}$ Isoform-Deficient Mice
}

\author{
Luis Polo-Parada, Christian M. Bose, Florian Plattner, and Lynn T. Landmesser \\ Department of Neurosciences, Case Western Reserve University, School of Medicine, Cleveland, Ohio 44106
}

\begin{abstract}
Mice that lack all three major isoforms of neural cell adhesion molecule (NCAM) (180 and $140 \mathrm{kDa}$ transmembrane, and $120 \mathrm{kDa}$ glycosylphosphatidylinositol linked) were previously shown to exhibit major alterations in the maturation of their neuromuscular junctions (NMJs). Specifically, even by postnatal day 30, they failed to downregulate from along their axons and terminals an immature, brefeldin A-sensitive, synaptic vesicle-cycling mechanism that used L-type $\mathrm{Ca}^{2+}$ channels. In addition, these NCAM null NMJs were unable to maintain effective transmitter output with high-frequency repetitive stimulation, exhibiting both severe initial depression and subsequent cyclical periods of total transmission failures that were of presynaptic origin. As reported here, mice that lack only the $180 \mathrm{kDa}$ isoform of NCAM downregulated the immature vesicle-cycling mechanism on schedule, implicating either the 140 or $120 \mathrm{kDa}$ NCAM isoforms in this important maturational event. However, 180 NCAM-deficient mice still exhibited many functional transmission defects. Although 180 NCAM null NMJs did not show the severe initial depression of NCAM null NMJs, they still had cyclical periods of complete transmission failure. In addition, several presynaptic molecules were expressed at lower levels or were more diffusely localized. Thus, the $180 \mathrm{kDa}$ isoform of NCAM appears to play an important role in the molecular organization of the presynaptic terminal and in ensuring effective transmitter output with repetitive stimulation. Our results also suggest that PKC and MLCK (myosin light chain kinase) may be downstream effectors of NCAM in these processes. Together, these results indicate that different isoforms of NCAM mediate distinct and important events in presynaptic maturation.
\end{abstract}

Key words: synaptic depression; FM1-43; vesicle cycling; neuromuscular transmission; MLCK; PKC

\section{Introduction}

The neural cell adhesion molecule (NCAM) exists in three major alternatively spliced isoforms, a $120 \mathrm{kDa}$ glycosylphosphatidylinositol-linked isoform and two 140 and $180 \mathrm{kDa}$ isoforms, with intracellular domains that differ only by the 266 amino acid long region that is spliced into the $180 \mathrm{kDa}$ isoform (Cunningham et al., 1987; Barbas et al., 1988). Mice lacking all three isoforms are viable (Cremer et al., 1994) but have recently been shown to exhibit multiple defects in neuromuscular maturation (Rafuse et al., 2000; Polo-Parada et al., 2001). Although neuromuscular junctions (NMJs) form and many presynaptic and postsynaptic molecules accumulate normally (Moscoso et al., 1998), these

\footnotetext{
Received June 3, 2003; revised Dec. 18, 2003; accepted Dec. 20, 2003.

This work was supported by National Institutes of Health Grants NS19640 and NS23678 from the National Institute of Neurological Disorders and Stroke. The mAb35 antibody was obtained from the Developmental Studies Hybridoma Bank under the auspices of the National Institute of Child Health and Human Development and maintained by the University of lowa Department of Biological Sciences. We thank Stephen Jones, Susann Brady-Kalnay, Stefan Herlitze, Corey Smith, Gartz Hanson, and Nenad llic for helpful advice on the experiments and this manuscript, and Katherine Lobur for her excellent care and genotyping of mice. Special thanks to Henry Tomasiewicz and Sue Dymecki, who generated the Ncam ${ }^{\text {tm1.1ciw }}$ mice while at the Emory University Cell Biology Department and the Carnegie Institute of Washington, respectively, and to John G. Wood, whose Grant AG11123 supported the development of the mice.

Correspondence should be addressed to Dr. Lynn Landmesser, Department of Neurosciences, Case Western Reserve University, 10900 Euclid Avenue, Cleveland, 0H 44106-4975. E-mail: It|@po.cwru.edu.

DOI:10.1523/JNEUROSCI.4406-03.2004

Copyright $\odot 2004$ Society for Neuroscience $\quad$ 0270-6474/04/241852-13\$15.00/0
}

NCAM-deficient NMJs fail to downregulate an immature transmitter release-vesicle-cycling mechanism that is brefeldin A (BFA)-sensitive and that uses L-type $\mathrm{Ca}^{2+}$ channels. In addition, stimulus-dependent synaptic vesicle exocytosis and endocytosis are not confined to active zones but occur along the entire preterminal axon and from regions of nerve terminal membrane that are not in direct contact with muscle. Furthermore, these junctions are unable to maintain transmitter output with repetitive stimulation, exhibiting both strong initial depression as well as periodic complete transmission failures at high but physiological stimulus rates. To begin to define how the absence of NCAM could contribute to these diverse defects in synaptic maturation, we analyzed, structurally and functionally, the process of synaptic maturation in mice lacking only the $180 \mathrm{kDa}$ isoform.

NCAM is abundantly expressed on developing myotubes and motor axons, and after synapse formation becomes confined to the NMJ, where it is expressed both presynaptically and postsynaptically as well as on Schwann cells (Covault and Sanes, 1986). Although NCAM can act as a homophilic adhesion molecule, the defects in synaptic maturation that we observed are more consistent with a role for presynaptic NCAM in helping to target and organize other presynaptic molecules via intracellular signaling cascades (Schmid et al., 1999; Paratcha et al., 2003) or direct protein-protein interactions. Furthermore, although homophilic interactions between presynaptic and postsynaptic 
NCAM could occur during the initial stages of NMJ formation, these should be precluded by the large width of the synaptic cleft at mature NMJs.

Mice lacking only the $180 \mathrm{kDa}$ isoform were previously shown to have defects in the development of the olfactory bulb (Tomasiewicz et al., 1993) and in circadian rhythms (Shen et al., 2000). The $180 \mathrm{kDa}$ isoform has also been suggested to play a role in synapse stabilization and rapidly accumulates at sites of contact between axonal growth cones and postsynaptic cells in hippocampal cultures (Sytnyk et al., 2002). We show here that mice lacking only the $180 \mathrm{kDa}$ NCAM isoform downregulate the immature transmitter release-synaptic vesicle-cycling mechanism at NMJs similar to wild type, implicating either the 140 or 120 $\mathrm{kDa}$ NCAM isoform in this process. However, 180 isoformdeficient NMJs, although failing to exhibit initial depression observed in NCAM-deficient NMJs, lacked paired-pulse facilitation. They also exhibited periodic total transmission failures and accompanying deficits in motor behavior. Together, these observations indicate that different isoforms of NCAM play distinct but critical roles in presynaptic maturation and function.

\section{Materials and Methods}

Mice. The total NCAM-deficient mice used, $\mathrm{Ncam} 1^{\mathrm{tm} 1 \mathrm{cgn}}$, was originally generated by Cremer et al. (1994) on a C57BL/6J background and was subsequently raised and bred locally. The 180 isoform-deficient mouse line used, Ncam ${ }^{\text {tml.1ciw }}$, differed from the 180 -deficient line $\mathrm{Ncam}^{\text {tmlcwr }}$, which was generated and described previously (Tomasiewicz et al., 1993). The Ncam ${ }^{\text {tmlcwr }}$ contains a targeted mutation in the Ncam gene where exon 18, which encodes sequences unique to the $180 \mathrm{kDa}$ isoform, is flanked by FLP recombinant targets that are recognized by the site-specific recombinase FLP. The Ncam ${ }^{\text {tm } 1 \mathrm{cwr}}$ mice were crossed with mice constitutively expressing FLP recombinase. As expected, some of the offspring from this cross were missing exon 18 in one copy of the NCAM gene, as determined by PCR (H. Tomasiewicz, personal communication). Following recently agreed on conventions, the resulting line was designated Ncam ${ }^{\text {tm1.ciw }}$. For each experiment, wild-type, heterozygote $(+/-)$, and homozygote $(-/-)$ mice were identified by PCR using primers that distinguish wild-type from mutant alleles (Cremer et al., 1994; Shen et al., 2000). Data presented for $+/+$ mice in this study are from the 180 deficient line unless noted otherwise. These did not differ in any obvious way from our previous observations made on $+/+$ mice from the line lacking all isoforms of NCAM.

Immunostaining. Mice were killed in a $\mathrm{CO}_{2}$ chamber, and the semitendinosus muscles were quickly removed and fixed with $3.7 \%$ formaldehyde (Sigma, St. Louis, MO) for 30 min, washed with 5\% sucrose in PBS, and cryoprotected in $30 \%$ sucrose for $24 \mathrm{hr}$. Frozen $(40 \mu \mathrm{M})$, sagittal, and cross $(14 \mu \mathrm{M})$ sections were cut and mounted on previously subbed glass slides, air-dried, and stored at $-70^{\circ} \mathrm{C}$. Alexa 488 - or 546 -conjugated $\alpha$-bungarotoxin (Molecular Probes, Eugene, OR) or monoclonal antibody (mAb) 35 antibody (Developmental Studies Hybridoma Bank, University of Iowa, Iowa City, IA) conjugated with Alexa 546 were used to visualize acetylcholine receptors (AChRs). Immunostaining was performed by blocking the sections with $2 \%$ BSA in PBS and incubating with the appropriate primary antibodies at $4^{\circ} \mathrm{C}$ for $24 \mathrm{hr}$, followed by incubation with fluorochrome-conjugated secondary antibodies (Zymed, San Francisco, CA) for $2 \mathrm{hr}$ at room temperature, washing in PBS and, finally, coverslipping the slides with Prolong Antifade (Molecular Probes). The fluorescent signal of the CW65 antibody (against $\mathrm{Ca}_{\mathrm{V}} 2.1 \mathrm{Ca}^{2+}$ channel) was further amplified using an Alexa Fluor 488 signal amplification kit for fluorescein (Molecular Probes). Images were digitally photographed $(40 \times)$ with an upright BX51WI Olympus (Tokyo, Japan) microscope equipped with an Olympus 2000 or Magnifier digital camera. Images were analyzed with the Metamorph Imaging System (Universal Imaging Corporation, West Chester, PA). To compare intensity of immunostaining between samples, all were stained using the same antibody concentrations and times, and quantification of pixel intensity was performed using the same set of image acquisition parameters. Any modifications to the acquired digital figures were limited to changes in color brightness and contrast using Adobe Photoshop 7.0 (Adobe Systems, San Jose, CA) or Corel Draw 11 (Corel, Ottawa, Ontario, Canada), and these were performed similarly when any comparisons were made between sections.

Antibodies. CW65 ( $\mathrm{Ca}_{\mathrm{v}}$ 2.1), RO25b (panNCAM polyclonal), and NCAM-13 (panNCAM monoclonal) were a generous gift from Maureen McEnery (Case Western Reserve University, Cleveland, OH), Urs Rutishauser (Memorial Sloan-Kettering Cancer Center, New York, NY), and Carl Lagenaur (University of Pittsburgh, Pittsburgh, PA), respectively. Synaptic vesicle 2 (SV2), mAb35 was obtained from Developmental Studies Hybridoma Bank, D3 (NCAM-180 monoclonal) from Deutsche Samm Lung von Mikroorganismen und Zelkulturen $\mathrm{GmbH}$ (German collection of microorganisms and cell cultures) (Braunschweig, Germany), RMO-270 anti-neurofilament $160 \mathrm{kDa}$ (NF-M) from Zymed, Rim fromTransduction Laboratories (Lexington, KY), Syntaxin from Sigma, Munc 13.1 from Synaptic Systems (Gottingen, Germany), panMunc13 from Transduction Laboratories, and myosin light chain kinase (MLCK), which recognizes both nonmuscle and muscle-specific isoforms (Sigma).

SDS-PAGE immunoblotting. Mice [postnatal day (P) 30] were killed in a $\mathrm{CO}_{2}$ chamber, and the brains were quickly removed. The hippocampus was dissected out and homogenized in HEPES extraction buffer (in mM: 25 HEPES, $150 \mathrm{NaCl}, 1$ EDTA) containing 1\% NP-40 and a protease inhibitor mixture (Roche Diagnostics, Mannheim, Germany). The solubilized protein concentrations were determined (BCA method; Pierce, Rockford, IL) and adjusted to $1-2 \mu \mathrm{g} / \mathrm{ml}$. To remove sialic acid, the extract was incubated for $1 \mathrm{hr}$ at $37^{\circ} \mathrm{C}$ with $0.08 \mathrm{U} / \mathrm{ml}$ Neuraminidase Type X (Calbiochem, San Diego, CA). SDS sample buffer containing dithiotheritol was added to each sample, and the proteins separated by SDS-PAGE according to the Laemmli method on a gradient 5-20\% gel. The proteins were transferred onto polyvinylidene fluoride membranes, which were immunostained with panNCAM (NCAM-13) and appropriate HRP-conjugated secondary antibodies, which were visualized with a chemiluminescence kit (ECL; Pierce).

Synaptosomal preparations were prepared from P30 wild-type hippocampal lysates by differential centrifugation as described previously (Whittaker, 1984). Briefly, mouse hippocampal tissue was homogenized in ice-cold lysis buffer (10 mm Tris, pH 7.4, $150 \mathrm{~mm} \mathrm{NaCl}, 1 \%$ Triton $\mathrm{X}-100,1 \mathrm{~mm}$ EDTA, $0.025 \% \mathrm{NaN}_{3}$ ). This homogenate was centrifuged at $1000 \times g$ to remove nuclei and large debris. This supernatant was then centrifuged at $10,000 \times g$ to obtain the crude synaptosomal fraction. Subsequently, this fraction was lysed hypo-osmotically for $30 \mathrm{~min}$ and centrifuged at $25,000 \times g$ to pellet a synaptosomal membrane fraction.

Intracellular recording. Semitendinosus muscles, with the nerve supply intact, were isolated and immediately placed into well oxygenated Tyrode's solution. The muscles were gently extended and pinned flat in a Sylgard (Dow Corning, Midland, MI)-coated recording chamber. The nerves were sucked tight into polyethylene-stimulating electrodes pulled from polyethylene tubing (PE-190; Clay Adams, Parsippany, NJ). Standard electrophysiological techniques were used to record miniature endplate potentials (MEPPs) and evoked endplate potentials (EPPs) in normal Tyrode's (125 mm NaCl, $5.37 \mathrm{~mm} \mathrm{KCl}, 24 \mathrm{~mm} \mathrm{NaHCO}_{3}, 1 \mathrm{~mm}$ $\mathrm{MgCl}_{2}, 1.8 \mathrm{mM} \mathrm{CaCl}_{2}$, and 5\% dextrose) containing $1 \mu \mathrm{M} \omega$-conotoxin GIIIB, which specifically blocks voltage-gated sodium channels in muscle, thereby preventing contraction (Alomone Labs, Jerusalem, Israel). Briefly, sharp glass electrodes (World Precision Instruments, Sarasota, FL) were pulled (20-40 MÙ resistance), filled with $3 \mathrm{M} \mathrm{KCl}$, and single muscle fibers were impaled near the motor endplate. The initial resting potentials were between -70 and $-85 \mathrm{mV}$ and usually remained stable throughout the duration of the experiments. Electrophysiological measurements were not recorded if the resting potential increased by $>15 \%$ of its original value. Potentials were recorded via an intracellular amplifier (World Precision Instruments) using Axoscope software (40 KHz sampling rate; Axon Instruments, Union City, CA). MEPPs (100-200) were recorded from each muscle fiber over a 2-4 min recording period. Single EPPs (recorded at $0.5 \mathrm{~Hz}$ for 3-4 min), paired-pulse EPPs, or trains of EPPs (5-200 Hz) were recorded in normal Tyrode's solution using short $0.2 \mathrm{msec}$ monophasic electrical stimuli isolated from ground with a Grass PISU6P stimulus isolation unit (Grass Instruments, West Warwick, RI). 
For variance-mean plot analysis, EPPs were recorded from single cells at different $\left[\mathrm{Ca}^{2+}\right]_{\mathrm{o}}(0.25-10 \mathrm{~mm})$ and constant $\left[\mathrm{Mg}^{2+}\right]_{\mathrm{o}}(1 \mathrm{~mm})$. Only cells with a resting membrane potential (RMP) less than $-65 \mathrm{mV}$ and change in RMP $<5 \mathrm{mV}$ during recording were considered. EPPs (200$350)$ were recorded at $1 \mathrm{~Hz}$ at each $\left[\mathrm{Ca}^{2+}\right]_{0}$. Size of EPPs was measured using Axoscope 8.0 (Axon Instruments). The variance and the mean of the EPPs were calculated using Origen 6.1 (OrigenLab Northampton, MA). Using equations proposed by Clements and Silver (2000), the variance-mean was plotted and fit with Sigma Plot 2.0 (Jandel Scientific, Corte Madera, CA). The probability of release at $2 \mathrm{~mm}\left[\mathrm{Ca}^{2+}\right]_{\mathrm{o}}$ was estimated from the degree of curvature from the curve fit at the corresponding $\left[\mathrm{Ca}^{2+}\right]$. Statistical analyses were performed using Student's $t$ test in Sigma Plot 2.0 (Jandel Scientific).

Perineural recordings. At mammalian NMJs, the action potential does not actively propagate into the nerve terminal, and current from the last node depolarizes the nerve terminal electrotonically. By appropriate positioning of sharp electrodes, it is possible to record electrotonically conducted currents from the nerve terminal as voltage drops across the perineurium. As previously described in detail (Brigant and Mallart, 1982; Urbano et al., 2002), we used such perineural recordings to distinguish the sodium, potassium, and calcium currents occurring in the presynaptic terminal with trains of stimuli. Two negative current peaks were recorded after each stimulus, which corresponded to the $\mathrm{Na}^{+}$and $\mathrm{K}^{+}$currents, respectively. When the $\mathrm{Ca}^{2+}$-activated $\mathrm{K}^{+}$current was blocked with $100 \mu \mathrm{m}$ 3,4-diaminopyridine, a positive peak was revealed. This could be blocked entirely by $\mathrm{Cd}^{2+}$ and corresponds to the $\mathrm{Ca}^{2+}$ influx in the nerve terminal (Brigant and Mallart, 1982). Such currents were recorded in wild-type and 180 null NMJs to determine whether alterations in endplate currents, especially the influx of $\mathrm{Ca}^{2+}$, could account for the transmission failures seen with high-frequency repetitive stimulation at 180 NCAM null NMJs.

$\mathrm{N}$-(3-triethylammoniumpropyl)-4-(4-(dibutylamino)styryl) pyridinium dibromide optical imaging to visualize vesicle cycling. Endplates were labeled by incubation with the ACh receptor antibody (mAb35; Developmental Studies Hybridoma Bank) conjugated with Alexa 546 (Molecular probes) for $1 \mathrm{hr}$. This mAb binds to the nictotinic AChRs but does not block transmission. The synaptic vesicles within them were then loaded with $N$-(3-triethylammoniumpropyl)-4-(4-(dibutylamino)styryl) pyridinium dibromide (FM1-43) by stimulating the muscle nerve for 10 min at $10 \mathrm{~Hz}$, whereas the muscle was bathed in normal saline containing $12 \mu \mathrm{M}$ FM1-43. These parameters produced optimal loading of dye. After loading, the preparation was washed with high $\mathrm{Mg}^{2+}(12 \mathrm{~mm})$ low $\mathrm{Ca}^{2+}(0.5 \mathrm{~mm})$ Tyrode's for $10 \mathrm{~min}$ to remove the dye from the solution. Images were then captured with a $60 \times$ water immersion objective on an Olympus BX51W microscope by means of an Olympus 2000 digital camera connected in a computer (Dell Pentium V350; Dell Computer Company, Round Rock, TX). A neutral density filter was used to reduce light damage and to ensure that the camera was within its linear range. At the same time, a second image was captured to show mAb35-Alexa 546 labeling to demonstrate overall endplate morphology. The preparation was then returned to normal saline $\left(1.8 \mathrm{mM} \mathrm{Ca}^{2+}\right.$ and $\left.1 \mathrm{~mm} \mathrm{Mg}^{2+}\right)$ for 5 min, and images were captured every $10 \mathrm{sec}$ during various stimulation protocols. Images were stored digitally and were later color coded for pixel intensity using the Metamorph Image Analysis System. Montages and labeling of the psuedo-colored images were created using Corel Draw 11. Loss of dye with time after trains of stimuli at different frequencies allowed us to monitor synaptic vesicle exocytosis via the loss of FM1-43 fluorescence. Statistical analysis and graphs were performed using Origen 7.0 Graphs or Sigma Plot 3.0.

Behavioral studies. Mice were placed on a rotorod, which was then rotated at speeds of 4, 20, and $40 \mathrm{rpm}$, and the time until they fell off the rod was measured. Each mouse was tested four to five times at $4-5 \mathrm{~min}$ intervals. Data was obtained from three to six $180(-/-)$ or $(+/+)$ mice.

\section{Results}

The 180 NCAM-deficient mouse lacks the $180 \mathrm{kDa}$ isoform of NCAM, but the 140 and $120 \mathrm{kDa}$ isoforms are still expressed The mouse line used that lacked only the $180 \mathrm{kDa}$ isoform of NCAM was generated by $\mathrm{H}$. Tomasiewicz and S. Dymecki
(NIEHS Murine and Freshwater Biomedical Sciences Center, University of Wisconsin - Milwaukee Great Lakes WATER Institute, Milwaukee, WI) (Shen et al., 2000). We first confirmed, using P30 hippocampal lysates, that this mouse lacked only the $180 \mathrm{kDa}$ isoform of NCAM. As observed by the Western blots shown in Figure $1 A$, and in contrast to the total NCAM knock out $(\mathrm{KO})$, which we characterized previously (Polo-Parada et al., 2001 ), only the $180 \mathrm{kDa}$ isoform was missing. However, in addition, expression of the remaining 140 and $120 \mathrm{kDa}$ isoforms was clearly upregulated. Immunostaining of P30 endplates (Fig. 1 B) showed that NCAM staining was absent from the total NCAM KO but was still present in the 180 -specific KO. Consistent with the biochemical experiments using hippocampal lysates, endplate immunostaining also indicated that the expression of the remaining NCAM isoforms had been moderately upregulated. This increase was quantified and shown to be significant (Fig. 1C) $(p<0.005)$. Thus, although overall NCAM levels were not reduced below wild-type levels, the 180 isoform was specifically lacking.

\section{The immature vesicle-cycling-transmitter release system is downregulated in NCAM 180-deficient NMJs as in wild type, but vesicle cycling is not confined to presynaptic active zones apposed to muscle}

Before contact with target myotubes, motor axons in Xenopus cultures have been shown to release transmitter (Sun and Poo, 1987) and to cycle synaptic vesicles (Dai and Peng, 1996) along the length of the axon and growth cone. This process uses different molecular machinery for vesicle budding than the vesiclecycling process at the mature synapse and can thus be blocked by brefeldin A (Zakharenko et al., 1999). After myotube contact, this immature form of vesicle cycling is shut down along the soma and axon (Chow and Poo, 1985; Yao et al., 2000), and the release machinery becomes selectively targeted to the presynaptic active zones, which are precisely aligned with postsynaptic junctional folds (for review, see Sanes and Lichtman, 1999). Associated with this targeting of release machinery to the synapse, during in vivo development of NMJs in both chick (Dahm and Landmesser, 1991) and mouse (Lupa and Hall, 1989), synaptic vesicle antigens are gradually lost from the axons and, by late embryonic stages, become highly enriched at the terminal. A striking alteration in NMJs lacking all NCAM was their failure to downregulate the immature vesicle-cycling process along the axon (Polo-Parada et al., 2001). To determine whether this immature mechanism was also abnormally maintained in the 180-deficient junctions, we compared stimulation evoked uptake of the styryl dye FM1-43 at P30 NMJs from wild-type mice and those lacking all or only the $180 \mathrm{kDa}$ isoform of NCAM.

In wild-type junctions, uptake of FM1-43 occurred only in the presynaptic terminal (Fig. 2a, bottom, left panel) overlying the endplate, which was visualized with the anti-ACh receptor antibody mAb35 as shown at top. In contrast, and as reported previously (Polo-Parada et al., 2001), dye uptake into the -INMJ (middle panel) occurred both in the presynaptic terminal overlying the endplate as well as in hot spots along the entire length of the preterminal axon (arrows). In the 180-deficient NMJs, as shown by the example in the right panel, FM1-43 uptake did not occur along the length of the preterminal axon. Thus, in contrast to the mouse lacking all NCAM, the 180-deficient mice appear to shut down this immature cycling process normally. The total vesicle pool that could be labeled with FM1-43 was also not significantly different in size from the wild-type pool 


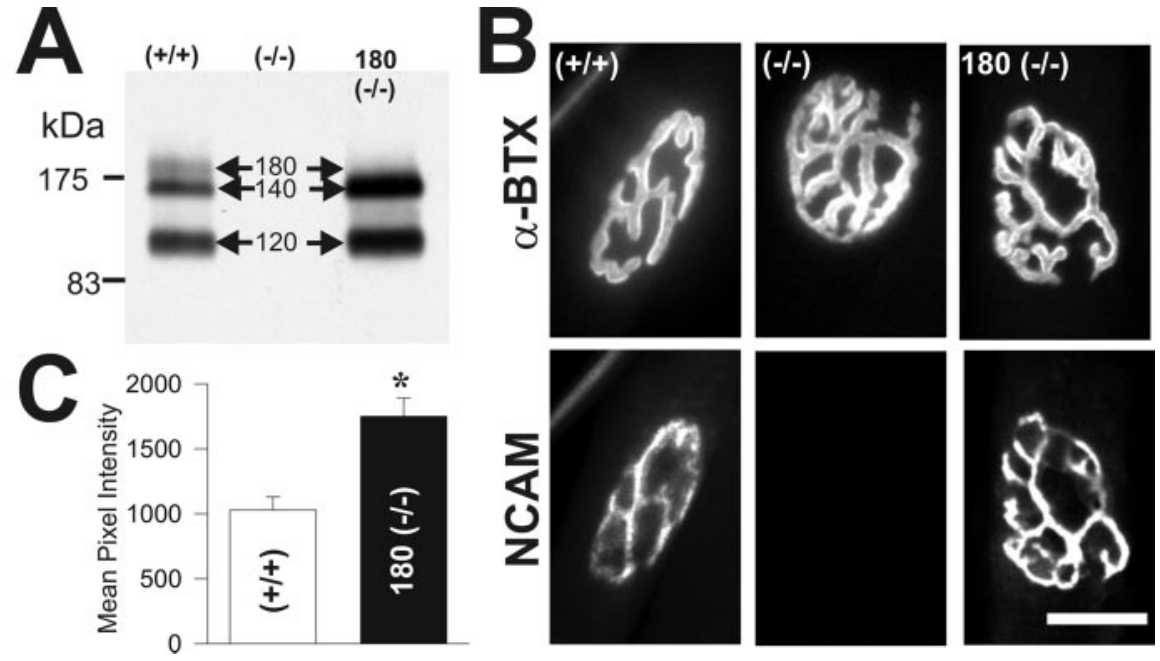

Figure 1. The distribution and expression levels of NCAM isoforms in a mouse that lacks only the 180 isoform of NCAM. $A$, Western blot of hippocampal lysates from wild-type mouse (left lane) lacking all isoforms of NCAM (middle lane) and lacking only the 180 is oforms of NCAM (right lane) stained with the NCAM 13 antibody that recognizes all isoforms of NCAM. All NCAM bands are lacking from the total KO mouse, whereas only the 180 band is lacking from the $180 \mathrm{KO}$ mouse. As shown in this example, however, the 120 and 140 isoforms were consistently upregulated in the 180 isoform-deficient mouse. Equal amounts of protein were loaded onto each lane and several other molecules; for example, $\beta$-tubulin did not differ in level (data not shown). $B$, Immunostaining of P30 NMJs with the pan-NCAM antibody R025b (bottom) and rhodamine $\alpha$-BTX to visualize endplate (top) also reveals the absence of NCAM in the total KO but a similar distribution to wild type in the NMJs of the 180 isoform-specific KO. However, as observed in the hippocampal lysates, the level of NCAM in the 180-deficient NMJs was elevated compared with wild type, and this was statistically significant $(p<0.005)$ when quantified, as shown in the bar graphs in C. Scale bar, (in B) $40 \mu \mathrm{m}$.

as shown by the quantification of fluorescence intensity (Fig. 2 B). Furthermore, the uptake of FM1-43 was not sensitive to BFA or the L-type $\mathrm{Ca}^{2+}$ channel blocker nifedipine but was completely blocked by the P/Q-type $\mathrm{Ca}^{2+}$ channel blocker $\omega$-agatoxin TK (Fig. 2C). Furthermore, as we described previously for NMJs lacking all NCAM, transmission measured electrophysiologically was mediated entirely by $\mathrm{P} / \mathrm{Q}$-type $\mathrm{Ca}^{2+}$ channels and was therefore completely blocked by $\omega$-agatoxin TK and was unaffected by L-type channel blockers. Thus, similar to wild-type synapses, the vesicle cycling at 180 -deficient NMJs appears to be mediated by entry of $\mathrm{Ca}^{2+}$ via P/Q-type channels (Urbano et al., 2002) and by clathrin-adaptor protein 2-mediated endocytosis, which is not sensitive to BFA (Brodin et al., 2000). Together, this data indicates that the developmentally regulated shutdown of the immature release machinery is regulated by either the 120 or 140 isoform of NCAM.

However, in the 180-deficient NMJs, we always detected FM1-43 uptake from a small region of the axon immediately adjacent to the terminal proper (Fig. $2 \mathrm{~A}$, right bottom, box) (the intensity of label in this region was usually less than at the endplate; thus, the region within the box is shown in the inset below with the brightness increased). This region also stained positively for the synaptic vesicle protein SV2 (Fig. 2D, arrow), whereas SV2 staining at wild-type junctions was always precisely confined to the endplate proper. The uptake of FM1-43 in this region was mediated by the mature vesicle-cycling process, because it was blocked by P/Q-type channel blockers but not by BFA (data not shown). We propose that this indicates that, similar to NMJs lacking all NCAM, vesicle cycling occurs from all portions of the nerve terminal and is not confined to presynaptic membrane overlying muscle (Polo-Parada et al., 2001).

This idea is supported by the fact that complete loading and unloading of dye in response to stimulation was faster in the 180-deficient compared with wild-type NMJs (Fig. 2E). This would be expected if vesicle cycling in 180deficient NMJs was occurring from all regions of the nerve terminal and was not confined only to active zones overlying muscle. Although faster dye loading-unloading could also occur if the quantal content of individual EPPs was increased, as shown later (Fig. $3 C$ ), there was no difference in quantal content between wildtype and 180-deficient NMJs. Faster kinetics of dye uptake and release was also observed in junctions lacking all NCAM (Polo-Parada et al., 2001). Finally, the NCAM 180-deficient NMJs exhibited another property of NMJs lacking all NCAM. In contrast to wild-type synapses, in which FM1-43 uptake in the absence of nerve stimulation is very low, FM1-43 uptake occurred without stimulation at 180deficient NMJs at normal extracellular $\mathrm{Ca}^{2+}$ levels (Fig. 2F), and although the magnitude was much less than with stimulation, it was appreciable (an average pixel intensity of $\sim 75$ compared with 1500 for nerve stimulation at $10 \mathrm{~Hz}$ for $10 \mathrm{~min}$ ). The degree of vesicle cycling in the absence of stimulation was similar to that observed in NMJs lacking all NCAM (Polo-Parada et al., 2001).

P30 NMJs lacking 180 NCAM fail to exhibit paired-pulse facilitation at normal transmitter release levels and have an altered sensitivity to extracellular $\mathrm{Ca}^{2+}$

To evaluate the properties of synaptic transmission at levels of transmitter release that would be expected in vivo, we recorded from junctions in normal $\mathrm{Ca}^{2+}$ and $\mathrm{Mg}^{2+}$ by using $\omega$-conotoxin GIIIB to block selectively $\mathrm{Na}^{+}$channels in the muscle or by using a cut muscle preparation, thereby preventing contraction. Under these conditions, MEPP amplitude and frequency did not differ significantly between $(+/+),(-/-)$, and $180(-/-)$ junctions (Fig. $3 A, B)$. To calculate the quantal content or number of vesicles released to a single stimulus, we divided the mean EPP amplitude by the mean mepp amplitude for each recorded cell. This method provides a more accurate estimation of quantal content than that derived from the coefficient of variation of EPP amplitude because of alterations in the statistical parameters of release in NCAM-deficient junctions, as reported previously (PoloParada et al., 2001). The mean number of quanta released by single stimuli (Fig. 3C) did not differ significantly between wild type and NCAM $180(-/-)$ null junctions, but both of these differed from NMJs lacking all NCAM, which had somewhat higher quantal contents [wild type, $86 \pm 6, n=8$; NCAM 180 $(-/-), 92.4 \pm 11, n=12$; NCAM $(-/-), 115 \pm 8, n=10 ; p<$ $0.05]$. As noted previously, transmission at 180-deficient synapses was insensitive to BFA and to L-type channel blockers but was completely blocked by the P/Q-type channel blocker $\omega$-agatoxin TK (data not shown) and thus, in this respect, did not differ from transmission at wild type or NMJs lacking all NCAM.

However, unlike wild-type synapses, which continued to show paired-pulse facilitation at normal levels of transmitter release, the NCAM $180(-/-)$-deficient synapses failed, as did the NCAM (-/-) NMJs (Polo-Parada et al., 2001), to exhibit paired-pulse facilitation at 10,8 , and $6 \mathrm{msec}$ intervals; at the 4 
msec interval, they in fact exhibited depression (Fig. 3D,E). NCAM 180 NMJs also differed from wild type in their sensitivity to extracellular $\mathrm{Ca}^{2+}$.

We characterized the $\mathrm{Ca}^{2+}$ dependence of the release process by determining the mean EPP amplitude from a single NMJ with extracellular $\mathrm{Ca}^{2+}$ concentrations ranging from 0.25 to $10 \mathrm{~mm}$. As described previously (Dodge and Rahamimoff, 1967; Polo-Parada et al., 2001), we found that for the $(+/+) \mathrm{NMJ}$, the EPP amplitude continually increased as $\mathrm{Ca}^{2+}$ levels rose between 0.25 and $10 \mathrm{~mm}$. In contrast, and as in the NCAM (-/ - ) NMJ (Polo-Parada et al., 2001), the EPP amplitude in the NCAM $180(-/-)$ NMJs had already reached near maximal levels at the physiological $\mathrm{Ca}^{2+}$ level of $2 \mathrm{~mm}$ and did not increase appreciably at higher levels of $\mathrm{Ca}^{2+}$ (Fig. 3F). One possibility for this finding could be that the ability of the 180 $-/-$ presynaptic terminal to release transmitter is already maximal at $2 \mathrm{mM} \mathrm{Ca}^{2+}$. Consistent with this idea, we found that using a variance-mean analysis (Clements and Silver, 2000) the mean probability of release at $180-/-$ NMJs was $0.799(n=2)$ compared with a $p$ value of 0.34 at $+/+$ NMJs $(n=3)$. Also in contrast to wildtype NMJs, they exhibited a very steep relationship between EPP amplitude and $\mathrm{Ca}^{2+}$ level between 0.25 and $2 \mathrm{~mm}$. Another striking finding was that NCAM 180 $(-/-)$ NMJs did not follow typical binomial statistics. At low levels of $\mathrm{Ca}^{2+}$ (i.e., 0.25-0.5 mM), wild-type junctions had low mean quantal contents and exhibited the expected number of failures on the basis of binomial statistics. In contrast, NCAM 180 (-/-) NMJs had mean quantal contents of $<2$ at these $\mathrm{Ca}^{2+}$ levels, but they exhibited almost no failures similar to what we had reported previously for the NCAM (-/-) NMJs (Polo-Parada et al., 2001) (data not shown).

NCAM 180 isoform-deficient synapses are unable to sustain transmitter output with repetitive stimuli

At P30, wild-type, NCAM (-/-), and NCAM $180(-/-)$ junctions were stimulated in normal $\mathrm{Ca}^{2+}$ and $\mathrm{Mg}^{2+}$ with $1 \mathrm{sec}$ trains of stimuli at 10, 20, 50, 100, and $200 \mathrm{~Hz}$. Consistent with our previous findings (Polo-Parada et al., 2001), at $200 \mathrm{~Hz}$ stimulation, the wild-type NMJs showed facilitation at the beginning of the train and effectively sustained transmission with only modest depression until the end of the train (Fig. $4 A$, top trace). As also described previously, NMJs lacking all NCAM (Fig. 4A, bottom trace) exhibited both strong initial depression and subsequent periods of complete transmission failures. In contrast, NCAM $180(-/-)$ NMJs exhibited neither the facilitation normally observed in $(+/+)$ NMJs nor the initial severe depression
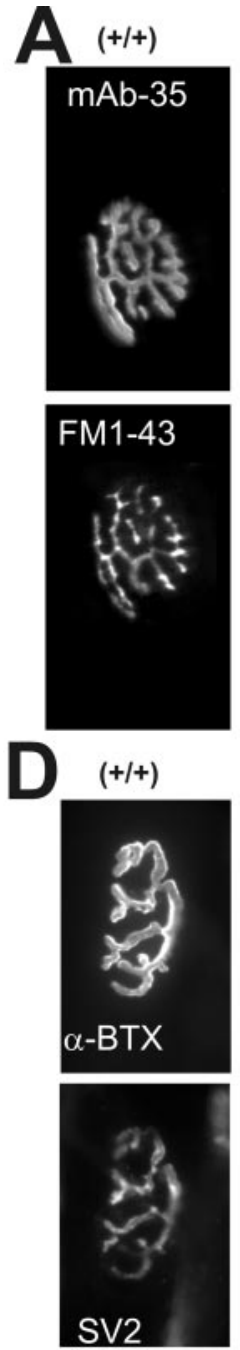
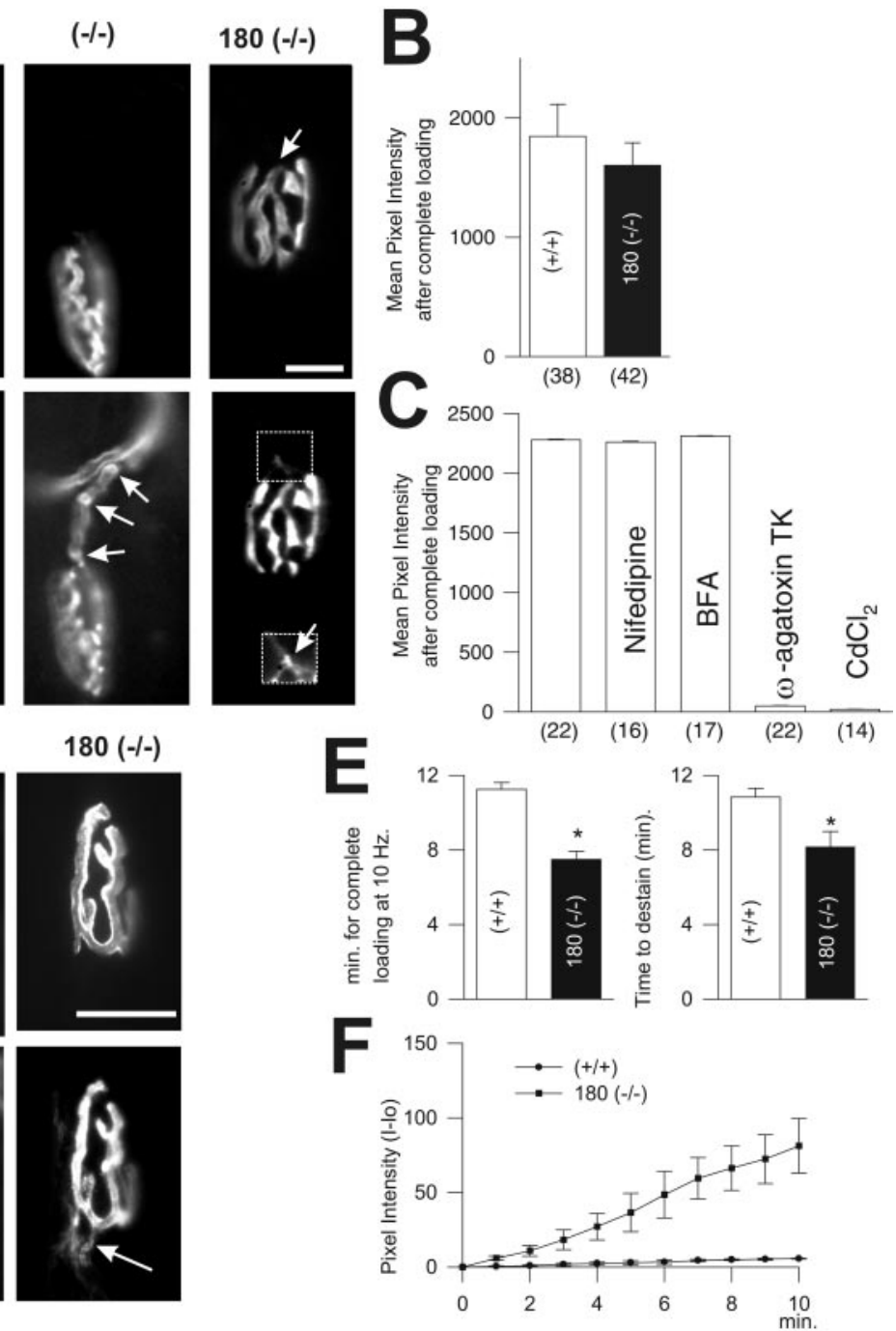

Figure 2. Characteristics of synaptic vesicle cycling and distribution at 180-deficient NMJs as revealed by FM1-43 and SV2 staining. A, FM1-43 uptake at 180-deficient P30 NMJs (bottom right) is primarily confined to the endplate as in wild-type NMJs (bottom left) and is absent from the entire length of the preterminal axon. Such staining along the length of the preterminal axon was previously observed in NMJs lacking all NCAM and is shown here in the bottom middle panel (arrows). However, FM1-43 uptake at 180-deficient NMJs was consistently observed from the axon immediately adjacent to the endplate (bottom right, box) where no corresponding ACh receptors were revealed with rhodamine $\alpha$-BTX (top left). Because staining in this region was always less intense than at the endplate proper, the contrast within this region has been digitally increased in the bottom inset (arrow). $B$, Overall level of staining after complete loading with FM1-43 did not differ significantly between wild-type and 180-deficient NMJs, indicating similarly sized vesicle pools. C, FM1-43 uptake in response to electrical stimulation was not affected by the L-type $\mathrm{Ca}^{2+}$ channel blocker nifedipine $(50 \mu \mathrm{M})$ or brefeldin $\mathrm{A}(10 \mu \mathrm{g} / \mathrm{ml})$ but was completely blocked by $\omega$-agatoxin TK (1 $\left.\mu \mathrm{M}\right)$ and cadmium chloride $(30 \mu \mathrm{M})$, indicating that vesicle cycling at these NMJs like that at wild-type mouse NMJs is mediated by calcium entering through P/Q-type channels. D, Immunostaining for the synaptic vesicle antigen SV2 bottom also shows staining of the axon immediately adjacent to the endplate proper in the 180-deficient NMJ (right, arrow), which was never observed in wild-type NMJs (left). E, Compared with wild type, the time it took to obtain both maximal staining and destaining was significantly faster at 180 -deficient NMJs $\left({ }^{*} p<0.005\right) . F$, In $2 \mathrm{~mm}$ extracellular $\mathrm{Ca}^{2+}$ and in the absence of stimulation, uptake at wild-type NMJs is minimal, whereas the 180-deficient NMJs exhibit appreciable uptake.

at the beginning of the train observed in NMJs lacking all NCAM (Polo-Parada et al., 2001). However, similar to the NCAM -/NMJs, at stimulus frequencies of $\geq 50 \mathrm{~Hz}$, they exhibited prolonged periods of complete transmission failure (Fig. $4 B$ ). This pattern of cyclical transmission failure was observed in all recorded NMJs and, although it varied somewhat from junction to junction, it was quite constant for a given junction. When the evoked response returned after a period of failures, the amplitude reached that of the initial EPP in the train. In this regard, it differed from NMJs lacking all NCAM, in which evoked re- 

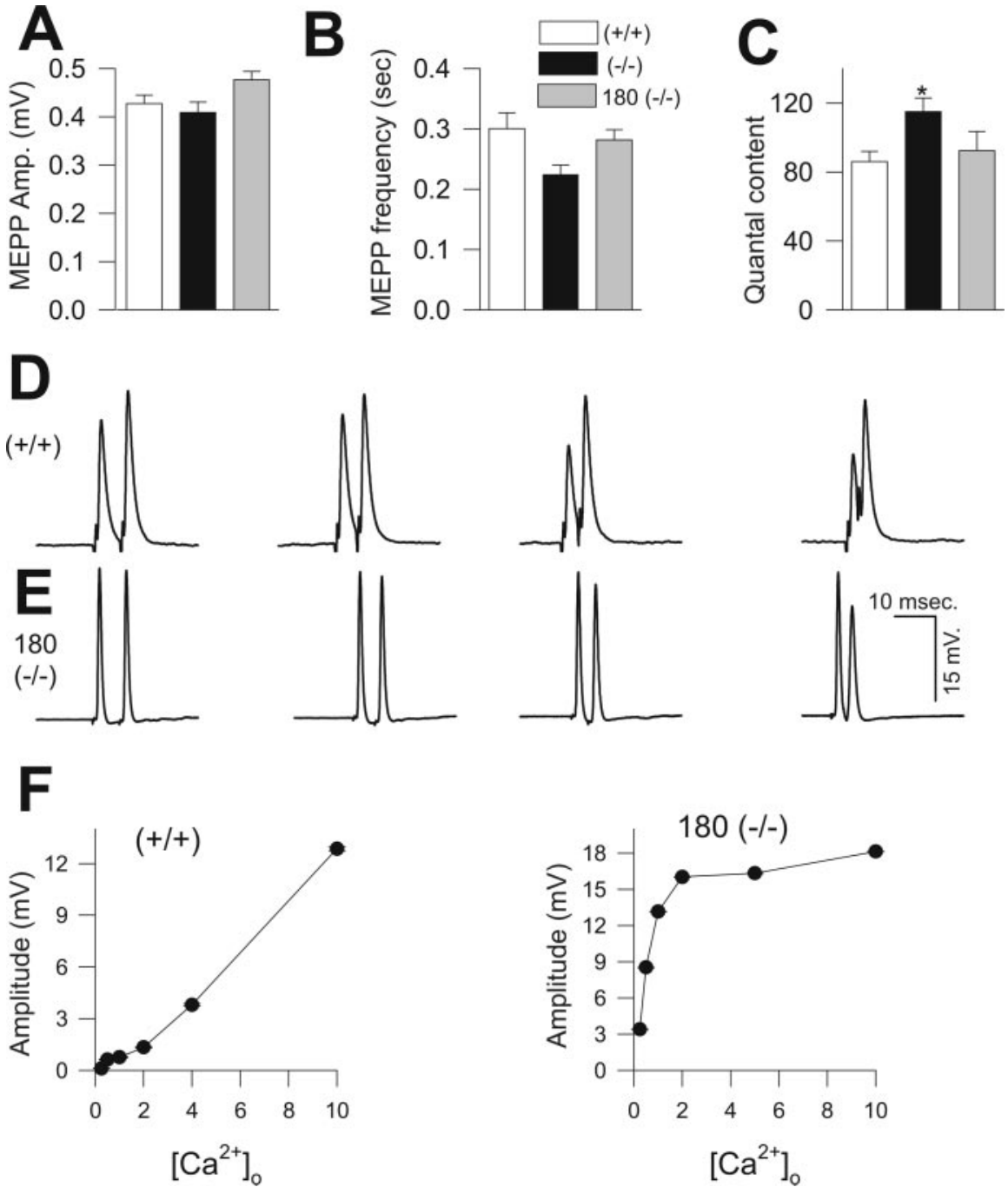

Figure 3. Transmitter release properties at P30 180-deficient NMJs compared with wild type and those that lack all NCAM. A, MEPP amplitude did not differ between junctions from wild-type $(+/+)$, NCAM-deficient $(-/-)$, or NCAM 180 isoformdeficient mice. B, MEPP frequency did not differ statistically between 180-deficient and wild-type junctions. As shown previously, it was moderately decreased in NCAM-deficient junctions. C, Quantal content in response to single stimuli also did not differ between 180 -deficient and wild-type NMJs. These values both differed significantly $\left({ }^{*} p<0.005\right)$ from NMJs lacking all NCAM whereas, as shown previously, quantal content was slightly elevated. $D$, Intracellular recordings illustrating that wild-type NMJs (top trace) exhibit paired-pulse facilitation at physiological levels of $\mathrm{Ca}^{2+}$ and $\mathrm{Mg}^{2+}$ at intervals of $10,8,6$, and $4 \mathrm{msec}$, whereas NCAM 180-deficient NMJs (bottom trace) do not. $F$, The relationship between extracellular $\mathrm{Ca}^{2+}$ levels and the amplitude of evoked EPPs for a single wild-type (left) and 180-deficient (right) NMJ.

sponses only returned to the steady state depressed level (Fig. $4 A$ ) In contrast to the NCAM $(-/-)$ NMJs, which only exhibited total transmission failures at higher frequencies $(200 \mathrm{~Hz})$, the 180-/- NMJs exhibited more failures at any given frequency (Fig. 4C), and these began to appear at frequencies as low as $20 \mathrm{~Hz}$ (Fig. $4 B$ ).

The data just presented strongly suggest that certain aspects of transmission are sensitive to the specific absence of the $180 \mathrm{kDa}$ isoform of NCAM. As shown in Figure 1, because of the upregulation of the 120 and $140 \mathrm{kDa}$, overall levels of NCAM were not reduced in the 180 isoform null NMJ. Additional evidence that the defects in transmission observed in the 180 NCAM null NMJs were not attributable to a reduction in overall NCAM levels is that no failures in transmission were detected with high-frequency repetitive stimulation in heterozygotes of the mouse line lacking all isoforms of NCAM (Fig. 4D). We confirmed that each isoform

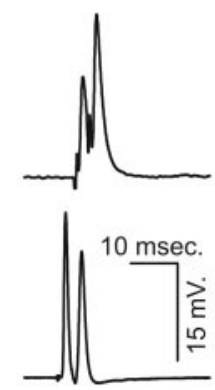

of NCAM was reduced in these heterozygotes by $\sim 50 \%$, as would be expected (data not shown).

The total transmission failures are not associated with alterations in nerve terminal currents, including the influx of $\mathrm{Ca}^{2+}$

At the mouse neuromuscular junction, the nerve terminal is not actively invaded by the action potential but is depolarized by currents that spread passively from the last node and preterminal portions of the axon. One possible explanation for the transmission failures in NMJs lacking all or only the $180 \mathrm{kDa}$ isoform of NCAM would be a block in the spread of such current so that the terminal membrane is not adequately depolarized. Alternatively, $\mathrm{Ca}^{2+}$ channels might be desensitized or altered in other ways so that $\mathrm{Ca}^{2+}$ influx would fail to occur even if the terminals were depolarized. To address these possibilities, we made perineural recordings from wild-type and $180 \mathrm{kDa}$ isoform null NMJs. When electrodes are appropriately positioned, it is possible to record extracellularly the currents from the nerve terminal membrane as voltage drops across the epineurium (Brigant and Mallart, 1982; Urbano et al., 2002). As shown in Figure $5 A$, this current can be resolved into two negative peaks, a fast initial peak that corresponds to sodium influx in the last heminode node and preterminal axon and a second, slower negative peak that reflects the $\mathrm{K}^{+}$current. When the $\mathrm{Ca}^{2+}$-activated $\mathrm{K}^{+}$current is blocked by the addition of 2,3,-diaminopyridine (Fig. $5 B$ ), a positive voltage deflection is revealed. This can be entirely blocked by $\mathrm{Cd}^{2+}$ and represents $\mathrm{Ca}^{2+}$ influx into the nerve terminal.

The nerve terminal sodium and potassium currents from a $180 \mathrm{kDa}$ isoform null NMJ are shown in Figure 5C during a 200 $\mathrm{Hz}$ train. Although numerous total failures in transmission were always detected within 5-10 stimuli at this repetition rate (Fig. $4 B$ ), we did not observe any complete blockage or even substantial reduction in the $\mathrm{Na}^{+}$or $\mathrm{K}^{+}$currents that would indicate the nerve terminal had failed to be depolarized. The modest reduction in the $\mathrm{K}^{+}$ current observed with high-frequency repetitive stimulation that is obvious in Figure $5 C$ also occurred in wild-type junctions. When 2,3,-diaminopyridine was applied to reveal the $\mathrm{Ca}^{2+}$ current, we also did not observe any large reduction in $\mathrm{Ca}^{2+}$ influx that could account for the transmission failures (Fig. 5D). As seen in this trace, the amplitude of the $\mathrm{Ca}^{2+}$ current peak was somewhat reduced, and the kinetics of the current slowed with highfrequency stimulation. However, when peak amplitudes from a number of endplates were quantified and displayed as a proportion of the initial response, no differences were observed between wild-type and $180 \mathrm{kDa}$ isoform null NMJs (Fig. 5E). Together, these results strongly suggest that the total transmission failures 
in the 180 isoform null NMJs are not caused by a lack of nerve terminal depolarization or, more importantly, by a reduction in $\mathrm{Ca}^{2+}$ influx. They point to, rather, some event downstream of $\mathrm{Ca}^{2+}$ influx, such as vesicle mobilization and dockingexocytosis as the explanation for these unusual transmission failures.

The involvement of PKC and MLCK in the alterations in transmission observed in NCAM-deficient NMJs

In several systems, phorbol esters such as PMA, which are capable of activating a number of PKC isoforms (for review, see Liu and Heckman, 1998), have been shown to enhance transmission by increasing quantal content (Minami et al., 1998; Hori et al., 1999; Berglund et al., 2002). We previously demonstrated that PMA could also prevent the total transmission failures observed in NMJs that lacked all isoforms of NCAM (PoloParada et al., 2001). As shown in Figure $6 \mathrm{~A}$, within tens of seconds of its application, PMA $(100 \mu \mathrm{M})$ also very effectively prevented the total failures of transmission observed in the 180 NCAM null NMJs. This amelioration of transmission was seen at all stimulus frequencies.

The effect of PMA in enhancing synaptic efficacy has been proposed to be caused by PKC activation (Stevens and Sullivan, 1998), which in turn promotes the more effective delivery of a sufficient number of synaptic vesicles to active zones, thereby allowing sustained transmission even at high stimulus repetition rates. However, it has recently been shown that PMA can also activate the DAG-sensitive phosphodiesterase Munc 13, and several studies have suggested that the effects of PMA on transmission are mediated by Munc-13 rather than by PKC activation (Betz et al., 1998; Searl and Silinsky, 1998; Rhee et al., 2002). Supporting the involvement of PKCs in our experiments, we found that brief preapplication of the specific PKC inhibitor Bisindolylmaleimide 1 (BIS-I) (Berglund et al., 2002) at $500 \mathrm{~nm}$ blocked the effect of PMA in preventing transmission failures in the 180 isoformdeficient synapses (Fig. $6 \mathrm{~B}$, bottom trace). This concentration of BIS-1 itself did not significantly increase the number of failures over that seen in the 180-deficient NMJ (Fig. 6 B, middle trace), at least over the time interval studied (40 $\mathrm{min}$ ).

Our previous study (Polo-Parada et al., 2001) suggested that the activation of MLCK, which is required for normal transmission (Mochida et al., 1994; Ryan 1999), might be deficient in NMJs that lacked NCAM and account for some of the deficits in transmission. We were led to this hypothesis by the fact that application of 1-(5-Cloronaphtalen-1-sulfonyl)-1H-hexahydro1,4-diazepine hydrochloride (ML-9) a specific inhibitor of MLCK, to P30 wild-type NMJs produced both the initial depression and subsequent total transmission failures observed in NMJs lacking all NCAM. When ML-9 was applied to the 180 NCAMdeficient NMJs, it produced a moderate increase in the number of failures (Fig. 6C). However, it also completely blocked the effect of PMA in preventing transmission failures. Together, these results suggest that functional MLCK is required for the targets that are activated by PMA, presumably via PKC, to prevent transmission failures. As shown in Figure $7 A$, staining with an antibody that recognizes both smooth muscle and nonmuscle isoforms of MLCK demonstrated that MLCK was expressed diffusely within the muscle fibers but was present at much higher levels in synaptic terminals. When the levels of junctional MLCK were quantified by the intensity of immunostaining (Fig. 7A, bar graph), we found that these were increased in the 180 NCAM-deficient synapses compared with wild-type NMJs. This might represent a compensatory mechanism in response to inadequate activation of MLCK in 180 NCAM null NMJs. 


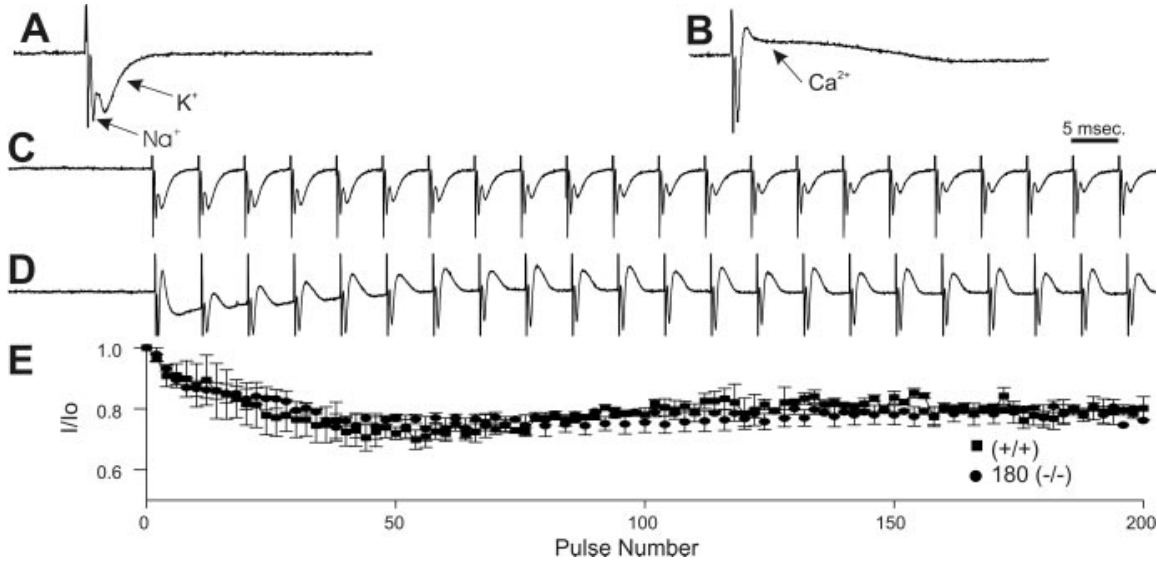

Figure 5. A, Epineural recordings of passively conducted $\mathrm{Na}^{+}, \mathrm{K}^{+}$, and $\mathrm{Ca}^{2+}$ currents from the nerve terminal during high-frequency stimulus trains). Appropriate placement of the electrode in the preterminal nerve reveals an extracellular current recording consisting of two negative peaks, the first corresponding to $\mathrm{Na}^{+}$influx and the second to $\mathrm{K}^{+}$flux (see Results for more details). These recordings from a $180 \mathrm{kDa}$ isoform null NMJ were indistinguishable from those recorded from wild-type junctions (Brigant and Mallart, 1982). B, After blockade of the $\mathrm{Ca}^{2+}$-activated $\mathrm{K}^{+}$current with $100 \mu \mathrm{m}$ 3,4,-diaminopyridine, an outward current that could be blocked by $\mathrm{Cd}^{2+}$ (data not shown) and that represents the $\mathrm{Ca}^{2+}$ influx at the nerve terminal is revealed. $C$, During a $200 \mathrm{~Hz}$ train from a $180 \mathrm{kDa}$ isoform null NMJ, there are no large reductions or block of either $\mathrm{Na}^{+}{ }^{+}$or K ${ }^{+}$currents (see Results for more details). $D, \mathrm{Ca}^{2+}$ currents, after blockade of the $\mathrm{Ca}^{2+}$-activated $\mathrm{K}^{+}$currents as above, recorded from a $180 \mathrm{kDa}$ isoform null NMJ during a similar train also do not reveal any large reductions or block in $\mathrm{Ca}^{2+}$ currents that could explain the total transmission failures (Fig. 4) seen at this stimulation frequency in these junctions. $E$, The amplitude of the peak $\mathrm{Ca}^{2+}$ currents for successive stimuli in $200 \mathrm{~Hz}$ trains relative to the first response in the train for wild-type (filled squares) and $180(-/-)$ NMJs reveals a moderate reduction in amplitude and lengthening of the time course, but no significant differences between $(+/+)$ and $(-/-)$ NMJs was detected. The slight slowing of the $\mathrm{Ca}^{2+}$ current was also observed in both types of synapses. Importantly, there were no total blockages or large reductions in $\mathrm{Ca}^{2+}$ currents that could account for the total transmission failures at $180 \mathrm{kDa}$ isoform null NMJs.

\section{Alteration in the expression of several presynaptic molecules in 180 NCAM-deficient synapses}

Similar to the mouse lacking all NCAM, in those NMJs lacking only the $180 \mathrm{kDa}$ isoform of NCAM, the expression and localization of several presynaptic molecules was altered. The expression of the calcium channel isoform $\mathrm{Ca}_{\mathrm{v}} 2.1$ was reduced and more diffusely localized (Fig. $7 B$ ). In addition, the expression of Rab 3-interacting molecule (RIM), a molecule involved in the modulation of transmission (Schoch et al., 2002), was also greatly reduced. Finally, the expression of syntaxin was also decreased (Fig. $7 B$ ). The alteration in the expression or targeting of such molecules could contribute to some of the functional defects in transmission that were observed at the 180 NCAM-deficient NMJs. However, it is important to note that alterations observed at the light microscope level, for example, in the distribution of the $\mathrm{Ca}_{\mathrm{v}} 2.1 \mathrm{Ca}^{2+}$ channel, may not provide the level of resolution needed to relate these alterations to the physiological changes observed. For example, although the level and distribution of the $\mathrm{Ca}_{\mathrm{v}} 2.1$ channel appears altered, what is relevant for transmission is the relationship of these channels to presynaptic active zones, which cannot be determined by light microscope immunostaining.

\section{Alterations in the morphology of NCAM 180-deficient synapses}

In addition to the molecular differences described above, the overall morphology of 180 NCAM null synapses was also altered. Although typical pretzel-shaped NMJs developed by P30 as visualized with rhodamine $\alpha$-BTX staining (Fig. $8 A$ ), compared with wild-type NMJs, the width of the synaptic gutters when viewed from above was much thinner. This was a very consistent finding as shown by the three separate examples and by the bar graph in Figure $8 B$, in which gutter width was quantified from longitudi- nal sections as shown in $A$. Transverse sections of similar endplates at the far right suggest that this may occur because the gutters are narrower but deeper in the 180 NCAM null junctions. Thus, the overall amount of contact between presynaptic and postsynaptic membranes may not differ, consistent with relatively normal numbers of synaptic vesicles exocytosed in response to single stimuli. However, it is interesting that difference in gutter width was not observed in NMJs lacking all NCAM.

\section{The distribution of overall NCAM compared with the $180 \mathrm{kDa}$ isoform}

Many antibodies against NCAM are directed against the extracellular domain and thus do not distinguish between the three main isoforms because their extracellular domains are essentially similar. Using a polyclonal antibody that recognizes all three isoforms of NCAM, we confirmed previous observations (Covault and Sanes, 1986) and found that, although NCAM on the nonsynaptic portions of $+/+$ muscle fibers had been downregulated by P30, moderate levels of NCAM continued to be expressed at both the endplate and preterminal axon (Fig. 9A, left). In NMJs lacking all NCAM, staining with this antibody was absent (data not shown). However, it persisted in both endplate and preterminal axon in the 180 isoformdeficient NMJs (Fig. 9A, right), indicating that there was no obvious alteration in the distribution of the 140 and $120 \mathrm{kDa}$ NCAM isoforms when viewed together. However, we did note a consistent moderate increase in the level of staining, suggesting that the other two isoforms had been upregulated in the $180 \mathrm{kDa}$ isoformdeficient mouse. Although the small proportion of synaptic material in muscle precluded confirmation of this observation via Western blotting, the Western blot from hippocampal lysates from wild-type and 180 isoform-deficient mice shown in Figure $1 \mathrm{~A}$ confirmed that these isoforms were in fact upregulated. Although the overall levels of NCAM were elevated at 180deficient NMJs, its distribution, as shown in the enlarged examples at the right, appeared to be similar to wild type with a generally uniform distribution.

In contrast to the rather uniform distribution of overall NCAM, an antibody specific for only the $180 \mathrm{kDa}$ isoform exhibited a much more punctate staining pattern within the endplate of wild-type NMJs (Fig. 9C, left panel, top and middle rows), and such staining was absent from the 180-deficient NMJs. Although some staining was also detected in the preterminal axon near the endplate, we did not observe consistent staining along the length of the axon. Thus, the 180 isoform appears to be preferentially expressed at the synapse. Localization of the 180 isoform in transverse sections (Fig. 8C, right panel) also indicated its synaptic localization. A careful comparison with rhodamine $\alpha$-BTX staining in the same sections indicated that some of the 180 NCAM isoform was located in the presynaptic terminal. However, without immuno-EM localization, it is not possible to exclude that the $180 \mathrm{kDa}$ isoform is also located postsynaptically. Although this isoform is often considered to be neuron specific, it has been 
detected in cultured avian myotubes and in developing mouse muscle (Lyons et al., 1992; Rafuse and Landmesser, 1996). Western blots of P30 hippocampal lysates (Fig. 9B) also showed that the 180 and, to a lesser extent, 140 isoforms were enriched in the synaptosomal fraction (first lane) compared with the total hippocampal lysate (second lane).

NCAM 180-deficient mice exhibit motor defects consistent with transmission failures at high repetition rates

Mice lacking all NCAM, although motile and fertile, were found to have severe locomotor deficits that became apparent when they were tested on rotorods (Polo-Parada et al., 2001). Although they were indistinguishable from wild-type mice when the rods were rotated at low speeds, 20 and 40 $\mathrm{rpm}$, they were unable to stay on the rod for more than a few seconds, whereas wildtype mice could remain on the rods for $\sim 1$ min. Similar but even more severe deficits were detected in mice lacking only the 180 isoform of NCAM. The mean times that these mice were able to stay on the rod was $12 \pm 1.15 \mathrm{sec}$ at $10 \mathrm{rpm}$ and $5 \pm 0.93$ at 20 $\mathrm{rpm}($ mean \pm SEM). This differed significantly from wild-type mice, where all were able to remain on the rod for $60 \mathrm{sec}$ at both 10 and 20 rpm (Polo-Parada et al., 2001).

\section{Discussion}

As synapses form and mature, large arrays of presynaptic and postsynaptic molecules, which are required for effective transmission, become selectively distributed and organized at the synapse (Garner et al., 2002). Presynaptic maturation at the NMJ includes the selective targeting of transmitter release machinery to presynaptic active zones and the development of cellular and molecular mechanisms needed to sustain high levels of transmitter output with repetitive stimulation. We have shown previously that adult mice lacking all isoforms of NCAM maintain a BFA and L-type $\mathrm{Ca}^{2+}$ channel-sensitive, immature, vesicle-cycling mechanism along the length of the axon. They also exhibit multiple functional defects, including severe depression and total transmission failures with highfrequency repetitive stimulation (Rafuse et al., 2000; Polo-Parada et al., 2001) (Table 1). In mice lacking only the $180 \mathrm{kDa}$ isoform of NCAM as described here, the immature release mechanism was downregulated appropriately, indicating that either the 140 or120 $\mathrm{kDa}$ isoform plays a role in this important maturational step. However, numerous defects in transmission remained, suggesting that the $180 \mathrm{kDa}$ isoform of NCAM plays additional and distinct roles in preysnaptic maturation and function.
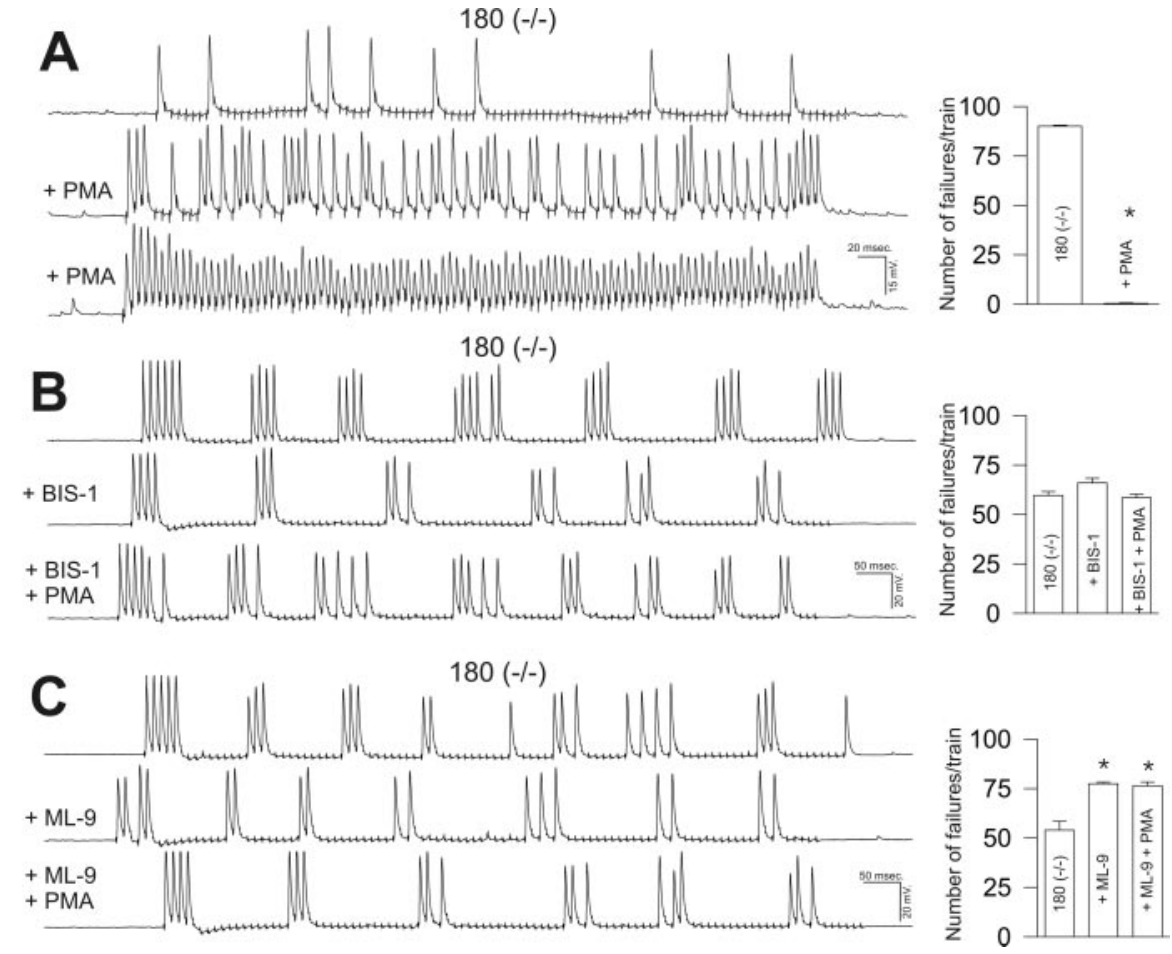

Figure 6. PKC and MLCK involvement in total transmission failures at 180 NCAM-deficient synapses. A, Application of $100 \mu \mathrm{M}$ PMA to a P30 180 null NMJ rapidly reverses the total transmission failures. The top trace is the untreated control, and the middle and bottom traces are 10 and $30 \mathrm{sec}$, respectively, after PMA application to the bath. The bar graph at the right indicates the number of failures per 1 sec $100 \mathrm{~Hz}$ train in untreated 180-deficient NMJs and after 30 sec of PMA application $(p<0.005)$. B, Application of the specific PKC inhibitor BIS-1 at $500 \mathrm{~nm}$ to another 180 null muscle did not increase the number of failures per $1 \mathrm{sec}$ train significantly (bar graph on right). However, it completely blocked the effect of PMA in reversing the transmission failures. C, Application to the bath of the MLCK-specific inhibitor ML-9 $(30 \mu \mathrm{M})$ increased to a moderate extent the number of failures per 1 sec train (middle trace and bar graph on right). However, it also completely blocked the effect of PMA in reversing the transmission failures (bottom trace and bar graph on right; $p<0.005$ ).
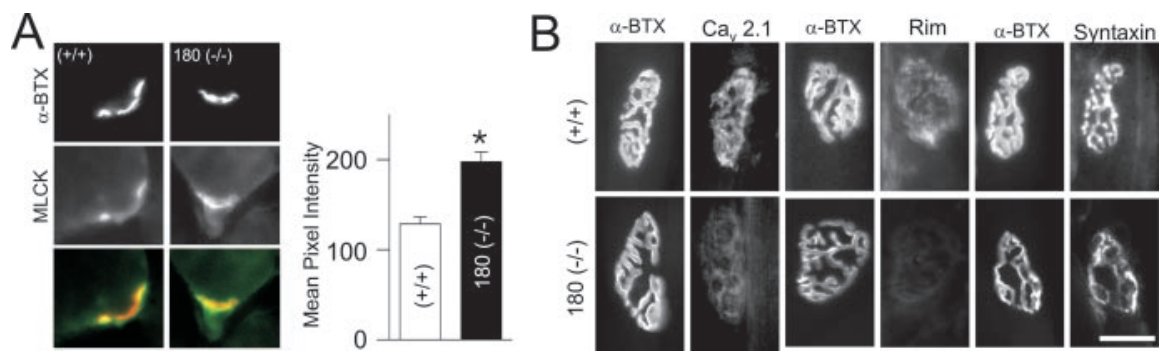

Figure 7. Distribution of synaptic molecules in 180-deficient and wild-type P30 NMJs. A, Immunostaining of $14 \mu \mathrm{m}$ transverse frozen sections from wild-type (left) and 180-deficient (right) NMJs with an antibody that recognizes both smooth muscle and nonmuscle isoforms of MLCK. Although weak diffuse staining (middle panel) was detected throughout the myotubes, much more intense staining was located at the junctions as defined by rhodamine $\alpha$-BTX staining shown in the top panel. The bottom panel shows a merged image with MLCK in green and $\alpha$-BTX in red. When the fluorescence intensity of the MLCK was quantified, the mean value from 50 junctions was significantly increased in the 180 deficient as compared with the control $(p<0.005)$. B, Several presynaptic molecules involved in transmission were also altered in level or distribution in the 180-deficient NMJ. Top row, Wild type; bottom row, 180-deficient NMJs. Each junction is presented as a pair of images with $\alpha$-BTX staining to define the endplate followed by staining for the $\mathrm{P} / \mathrm{Q} \mathrm{Ca}^{2+}$ channel subunit $\mathrm{Ca}_{\mathrm{v}} 2.1$, Rim, or syntaxin. All three exhibited weaker and more diffuse staining in the 180-deficient NMJ. Scale bar, $40 \mu \mathrm{m}$.

Downregulation of the immature cycling mechanism in $\mathbf{1 8 0}$ $\mathrm{kDa}$ NCAM isoform-deficient NMJs implicates the other NCAM isoforms in this process

Although immature neurons are able to release transmitter and cycle synaptic vesicles from their axons, soma, and growth cones, this immature form of transmission has been shown to be downregulated after contact with the postsynaptic cell in both Xenopus 

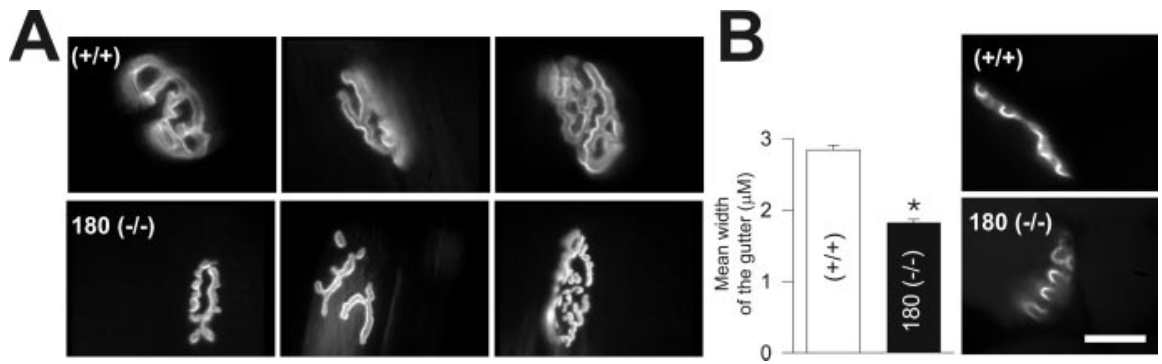

Figure 8. Morphological differences between 180-deficient and wild-type P30 NMJs. A, P30 endplates from wild-type (top) and 180 isoform-deficient (bottom) NMJs as visualized by staining with rhodamine $\alpha$-bungarotoxin. The width of the junctional gutter was consistently narrower in the 180-deficient endplates. In addition, although many exhibited pretzel-like shapes similar to wild-type endplates, in many cases, portions of the endplate, as visualized by postsynaptic rhodamine $\alpha$-bungarotoxin staining, were either disconnected or connected by very thin regions to the endplate proper (see middle panel for an example). Scale bar, $25 \mu \mathrm{m}$. B, Bar graph of the widths (mean \pm SEM) of the junctional gutters from 40 wild-type and 40180 NCAM null NMJs showing that the 180 null junctions were statistically less wide $\left({ }^{*} p<0.005\right)$. This apparent reduction in width, when endplates were viewed from above, may have resulted from the fact that the gutters were actually deeper when observed in transverse sections (far right). Scale bar, $10 \mu \mathrm{m}$.

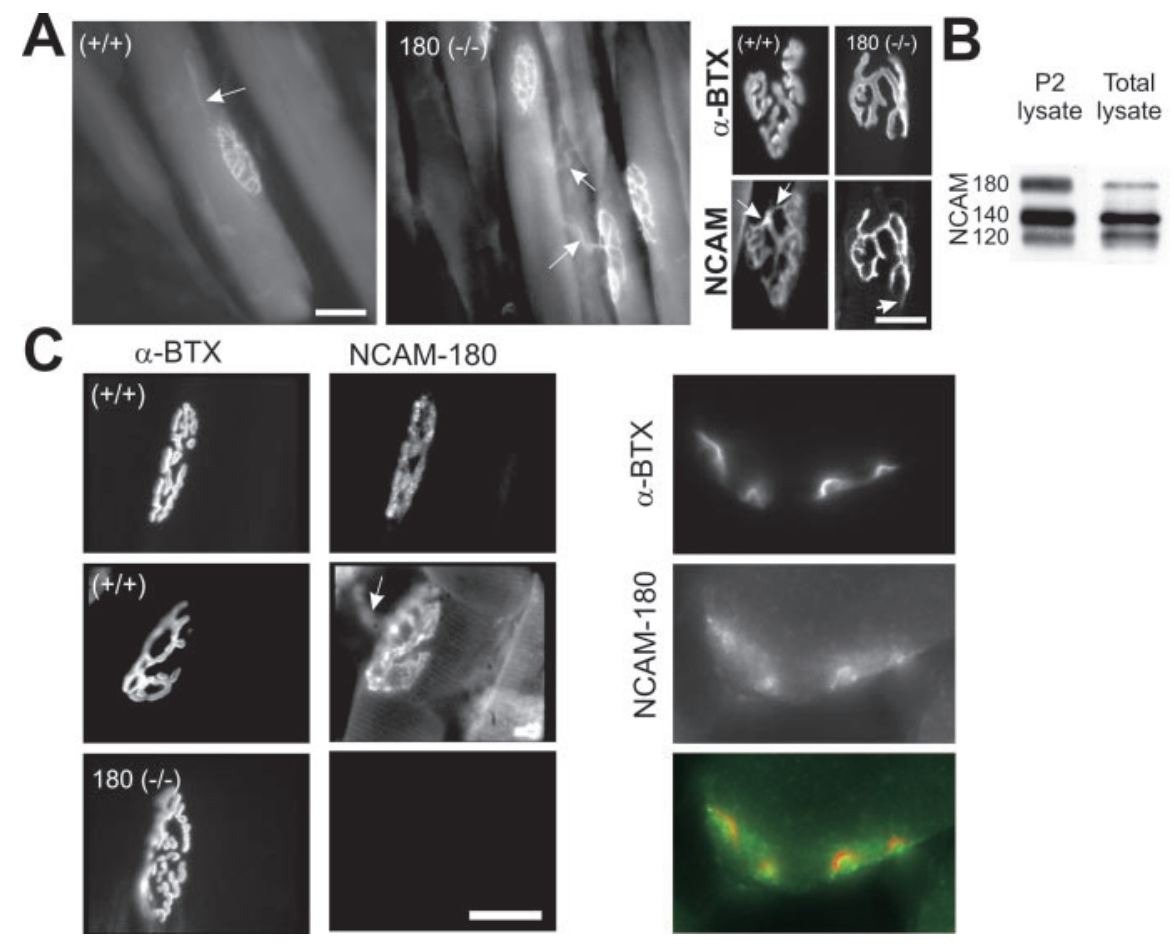

Figure 9. The distribution of overall NCAM in comparison with the 180 isoform in P30 NMJs. $A$, Left, The distribution of total NCAM expression at wild-type and 180 NCAM-deficient synapses as revealed by a polyclonal antibody (R025b) that recognizes all isoforms of NCAM. NCAM is expressed in both the endplate and at a somewhat lower level in the preterminal axon but is essentially absent from the nonsynaptic portions of the muscle fibers (staining here is not above background). Right, Higher magnification examples of pan NCAM staining showing NCAM (bottom) and rhodamine $\alpha$-bungarotoxin staining (bottom). There is relatively uniform staining throughout the endplate in both wild-type and 180 NCAM-deficient NMJs. The arrows point to staining of the preterminal axons in 180 null NMJs as they enter the endplate in a region where there are no underlying ACh receptors. $B$, Western blots stained with a pan-NCAM antibody from P30 wild-type hippocampal lysates. When compared with the total lysate (right band), the lysate from the synaptosomal fraction (left band) shows that the 180 isoform of NCAM and, to a lesser extent, the 140 isoform, are enriched at synapses. C, Staining with a monoclonal antibody that recognizes only the 180 isoform in P30 wild-type NMJs. Left panel, Endplates viewed from above (top and middle row) show that the 180 isoform has a more punctate staining pattern at the endplate, compared with overall NCAM. Although some much less intense staining was observed in the preterminal axon close to the endplate (arrow), overall axonal staining was weak to absent. 180 NCAM staining was absent from the 180deficient NMJ shown in the bottom panel. Right panel shows staining for 180 NCAM (middle row) in comparison with rhodamine $\alpha$-bungarotoxin staining (top row) in transverse sections of P30 wild-type muscles. The bottom row shows the merged image with rhodamine $\alpha$-bungarotoxin in red and 180 NCAM in green. Scale bars, $40 \mu \mathrm{m}$. and Drosophila neurons in culture (Chow and Poo, 1985; Zakharenko et al., 1999; Yao et al., 2000). Although the mechanisms underlying this downregulation are essentially unknown, it presumably involves some form of signaling from the postsynaptic cell. Because this process occurred in the 180 NCAM isoformdeficient mouse but not in mice lacking all NCAM, the presence of either the 140 or $120 \mathrm{kDa}$ isoform is sufficient to allow this process to occur. Whether this involves homophilic interactions between presynaptic and postsynaptic NCAM remains to be determined. NCAM-mediated adhesion between motor axon and myotube could simply allow other cell surface molecules to generate a signal. Alternatively, NCAM itself may be involved in such signaling events (Safell et al., 1997; Schmid et al., 1999; Kolkova et al., 2001; Koh et al., 2002; Paratcha et al., 2003). Whatever the precise cellular mechanism, the fact that this process fails to occur when all NCAM is absent places NCAM at a critical step in this process, even though synapses form and many other aspects of synaptic maturation take place.

\section{Alterations in transmission at $\mathbf{1 8 0}$ NCAM isoform-deficient NMJs}

When the immature form of vesicle-cycling-transmitter release was blocked pharmacologically at NMJs lacking all NCAM, the number of transmission failures with repetitive stimulation was reduced (Polo-Parada et al., 2001). This suggested that a competition between the immature and mature vesicle-cycling mechanisms for cellular components might at least partly explain the transmission failures. However, the present demonstration that the transmission failures still occurred in NMJs specifically lacking the 180 isoform of NCAM points to the lack of the 180 NCAM isoform as being the salient alteration, even though the immature cycling mechanism had been downregulated. This idea is reinforced by the fact that, because of the upregulation of the other two isoforms, overall NCAM levels were not reduced below wild type. This provides additional evidence that the transmission failures are attributable to the selective loss of the $180 \mathrm{kDa}$ isoform.

As in NMJs that lacked all NCAM, 180 $\mathrm{kDa}$ isoform-deficient NMJs had normal EPP amplitudes and quantal contents, showing that the basic machinery for exocytosis had assembled properly. Also, the $180 \mathrm{kDa}$ isoform-deficient NMJs did not exhibit depression with high-frequency repetitive stimulation, which we had previ- 
Table 1. Summary of transmission and vesicle cycling in wild-type, NCAM null, and 180 NCAM-deficient mice

\begin{tabular}{|c|c|c|c|c|}
\hline & $(+/+)$ & $(-/-)$ & $180(-/-)$ & Figure \\
\hline Quantal content & Normal & Increased & Normal & 3 \\
\hline MEPP (amplitude) & Normal & Normal & Normal & 3 \\
\hline MEPP (frequency) & Normal & Normal & Normal & 3 \\
\hline Paired-pulse facilitation & Yes & No & No & 3 \\
\hline $\mathrm{Ca}^{2+}$ dependency of transmitter release & Normal & Saturated at physiological $\mathrm{Ca}^{2+}(2 \mathrm{~mm})$ & Saturated at physiological $\mathrm{Ca}^{2+}(2 \mathrm{~mm})$ & 3 \\
\hline Depression at $50-200 \mathrm{~Hz}$ & Minimal & Extensive & Minimal & 4 \\
\hline Transmission failures & None & Many at $200 \mathrm{~Hz}$ & Many at $50-200 \mathrm{~Hz}$ & 4 \\
\hline Failures in AP propagation into the terminal & No & No & No & 5 \\
\hline $\mathrm{Ca}^{2+}$ channels responsible for transmission & P/Q-type & P/Q-type & P/Q-type & 2 \\
\hline $\mathrm{Ca}^{2+}$ channels involved in vesicle cycling (FM1-43) & P/Q-type & $\begin{array}{l}\text { 80\% L-type } \\
\text { 20\% P/Q-type }\end{array}$ & P/Q-type & 2 \\
\hline Vesicle cycling along the nerve & No & Yes & No & 2 \\
\hline $\begin{array}{l}\text { Kinetics of FM1-43 loading- unloading (with elec- } \\
\text { trical stimulation) }\end{array}$ & Control & Faster & Faster & 2 \\
\hline Vesicle loading without electrical stimulation & No & Yes & Yes & 2 \\
\hline Transmission sensitivity to BFA & No & No & No & 2 \\
\hline Vesicle cycling (FM1-43) sensitivity to BFA & No & Yes $(80 \%)$ & No & 2 \\
\hline Activation of PKC by PMA & Increase quantal content & Reduce number of failures & Reduce number of failures & 6 \\
\hline MLCK inhibition (ML-9) & Induces failures & Increases number of failures & Moderately increases number of failures & 6 \\
\hline NCAM levels & Control & Absent & NCAM 120 and 140 isoforms increased & 1 \\
\hline Distribution and levels of $P / Q$ Calcium channels & Control & Reduced and dispersed & Reduced and dispersed & 7 \\
\hline Rim immunostaining & Control & NA & Reduced & 7 \\
\hline Syntaxin immunostaining & Control & Reduced & Reduced & 7 \\
\hline Mean width of the synaptic gutter & Control & Similar to Control & Diminished & 8 \\
\hline
\end{tabular}

ously shown in NCAM-deficient NMJs of presynaptic origin (Polo-Parada et al., 2001). This suggests that one of the other isoforms of NCAM is required to prevent rundown of transmitter output with repetitive stimulation. However, similar to the NCAM null NMJs, those lacking only the $180 \mathrm{kDa}$ isoform, lacked paired-pulse facilitation, and their transmitter release had an altered sensitivity to extracellular $\mathrm{Ca}^{2+}$ with output saturating at physiological levels of $\mathrm{Ca}^{2+}$. The fact that the amplitude and time course of MEPPs did not differ between wild-type and 180 null NMJs, nor did the quantal content at physiological $\mathrm{Ca}^{2+}$ levels, make it unlikely that postsynaptic alterations such as saturation or desensitization of ACh receptors could explain these differences in paired-pulse facilitation and $\mathrm{Ca}^{2+}$ sensitivity. $\mathrm{Al}-$ ternatively, these could result from presynaptic changes in the sensitivity of the $\mathrm{Ca}^{2+}$ sensor or alternatively to alterations in $\mathrm{Ca}^{2+}$ entry and buffering, possibly because of changes in the number and distribution of $\mathrm{Ca}^{2+}$ channels or $\mathrm{Ca}^{2+}$ buffering organelles such as mitochondria. Our observation that the probability of release was increased at $180-/-$ NMJs is consistent with a presynaptic explanation. However, we cannot at present exclude some postsynaptic contributions to this phenomenon.

The most striking defect was the cyclical complete transmission failures with repetitive stimulation $>50 \mathrm{~Hz}$. Although these could be produced by failure of the action potential to depolarize the nerve terminal, extracellular current recordings from NCAM null NMJs, in which similar failures occurred, did not detect failures in nerve terminal depolarization (Polo-Parada et al., 2001). Here, we present additional data showing that adequate depolarization of the nerve terminal also occurs with high rates of repetitive stimulation of 180 NCAM-deficient NMJs. More importantly, we also demonstrated that there are no failures or large reductions in nerve terminal $\mathrm{Ca}^{2+}$ influx that could account for the total transmission failures observed. This suggests that events downstream of $\mathrm{Ca}^{2+}$ influx, such as an inability to deliver vesicles at rates needed to maintain transmission at high but physiological rates (i.e., $50 \mathrm{~Hz}$ ), may account for the transmission failures at
NCAM and 180 isoform NCAM NMJs. This could be attributable to alterations in the size of the pool of releasable vesicles (although this is not supported by our FM1-43 data for the 180 NCAM-deficient NMJs), to defects in mobilizing vesicles, or to subsequent steps in docking and fusion of vesicles at high rates.

\section{Vesicle mobilization, recruitment, priming, and the proposed involvement of PKC and MLCK in the NCAM 180 null phenotype}

The main functional defect in NCAM 180-deficient NMJs was an inability to maintain effective transmitter output with highfrequency stimulation. We hypothesize that this may be, in part, because of the fact that PKC and MLCK, which have been shown to play important roles in endocrine (Yu et al., 2000) and neural (Majewski and Iannazzo, 1998; Ryan, 1999) secretion, are not fully activated in the absence of NCAM 180. In neurons and chromaffin cells, trains of stimuli can activate PKCs, which help in maintaining transmitter output (Smith, 1999; Becherer et al., 2001). In some cases, this is thought to result from increasing the pool of releasable vesicles or its rate of refilling (Stevens and Sullivan, 1998; Berglund et al., 2002). Activated PKCs also translocate to the secretory surface (Saitoh et al., 2001), where they can phosphorylate proteins important in vesicle priming and exocytosis [for example, SNAP 25 (soluble $N$-ethylmaleimide-sensitive factor attachment protein)] (Nagy et al., 2002). Vesicle depletion from active zones and increased depression during trains of stimuli was produced by staurosporine application to frog NMJs (Becherer et al., 2001).

In NCAM 180-deficient NMJs, PMA prevented the total transmission failures, most likely through activation of PKCs, because its effect was completely prevented by the PKC-specific inhibitor BIS I. In addition to PKCs, Munc 13 isoforms can also be activated by phorbol esters and facilitate transmitter release (Rhee et al., 2002). However, BIS I did not antagonize the Munc 13-mediated increase in transmitter release in hippocampal cultures (Rhee et al., 2002), and the relative importance of Munc 13 
isoforms versus PKCs in enhancing transmitter release appears to vary with the type of synapse (Minami et al., 1998; Hori et al., 1999; Honda et al., 2000).

MLCK has also been shown to be important for normal transmission (Mochida et al., 1994; Ryan, 1999) and is thought to aid in the mobilization and delivery of vesicles to active zones. We found previously that the MLCK inhibitor ML-9, when applied to adult wild-type NMJs, phenocopied the transmission failures observed in NCAM null junctions (Polo-Parada et al., 2001). We showed here that inhibition of MLCK only moderately increased the number of failures at 180 NCAM-deficient NMJs, suggesting that MLCK activation was already impaired in the 180 NCAMdeficient NMJs. In both cases, MLCK inhibitors did not affect the size of the initial EPPs and only produced failures after a number of stimuli at high repetition rates. This suggests that MLCK at mouse NMJs may only be needed to mobilize and recruit vesicles at high levels of transmitter output. The fact that PMA had no effect in the presence of MLCK inhibitors suggests that the downstream targets of PKC activation that enhance transmitter release require normally active MLCK. Phosphorylation of myosin light chain, the only known substrate of MLCK (Kamm and Stull, 2001), is compatible with a role in vesicle mobilization. However, it will be important to determine the effects of PKC activators and MLCK inhibitors on presynaptic endplate currents, because these might be indirectly affected and contribute to the observed changes in transmitter release (Tokimasa, 1995). In summary, our results suggest that one or more isoforms of $\mathrm{PKC}$ as well as MLCK may not be properly activated in NMJs that lack the 180 $\mathrm{kDa}$ isoform of NCAM.

\section{Potential roles of 180 NCAM in presynaptic structure and function}

From the results presented here, it is clear that 180 NCAM is required for effective sustained transmission and that both $\mathrm{PKC}$ and MLCK are potential targets of NCAM-mediated signaling. We have shown that the $180 \mathrm{kDa}$ isoform of NCAM is enriched at the synapse, which might occur by its interaction with other synaptic proteins, for example, agrin, which is deposited in the synaptic basal lamina. In addition to its hypothesized role in ensuring the appropriate activation of PKC and MLCK, we have also shown that 180 NCAM is required to target synaptic vesicle cycling to active zones apposed to muscle and for the appropriate localization of a number of synaptic proteins such as RIM (Schoch et al., 2002) and P/Q-type calcium channels. NCAM 180 might achieve these roles by being part of a scaffold that organizes molecules that are important in transmission (Thomas et al., 1997). The 180 but not the $140 \mathrm{kDa}$ isoform of NCAM has been reported to bind to spectrin (Sytnyk et al., 2002). Furthermore, in Drosophila spectrin mutants, transmission, especially with repetitive stimuli, is impaired and a number of presynaptic proteins such as cysteine string protein, discs large, synapsin, and syntaxin are not appropriately localized (Featherstone et al., 2001). Using bioinformatic approaches, we identified highly conserved regions of the intracellular domain of NCAM and are currently screening for proteins that interact with these regions as well as for probing their roles in transmission.

Alternatively, NCAM may be part of one or more intracellular signaling cascades (Crossin and Krushel, 2000), and it has been reported previously to modulate neurite outgrowth via an FGF receptor-DAG-IP 3 pathway (Safell et al., 1997) or via an RASMAP kinase pathway (Schmid et al., 1999; Kolkova et al., 2001). Recently, it has been shown that the $140 \mathrm{kDa}$ isoform of NCAM can also function as a receptor for glial cell line-derived neuro- trophic factor and thereby affect axon growth and Schwann cell migration (Paratcha et al., 2003). Thus, our observations that different isoforms of NCAM mediate distinct aspects of presynaptic maturation add to these novel roles of NCAM and provide an amenable system to dissect out the different cellular pathways mediating these effects.

\section{References}

Barbas JA, Chaix JC, Steinmetz M, Goridis C (1988) Differential splicing and alternative polyadenylation generates distinct NCAM transcripts and proteins in the mouse. EMBO J 7:625-632.

Becherer U, Guatimosin C, Betz WJ (2001) Effects of staurosporine on exocytosis and endocytosis at frog motor nerve terminals. J Neurosci 21:782-787.

Berglund K, Midorikawa M, Tachibana M (2002) Increase in the pool size of releasable synaptic vesicles by the activation of protein kinase $\mathrm{C}$ in goldfish retinal bipolar cells. J Neurosci 22:4776-4785.

Betz A, Ashery U, Rickmann M, Augustin I, Neher E, Sudhof TC, Rettig J, Brose N (1998) Munc 13-1 is a presynaptic phorbol ester receptor that enhances neurotransmitter release. Neuron 21:123-136.

Brigant JL, Mallart A (1982) Presynaptic currents in mouse motor endings. J Physiol (Lond) 333:619-636.

Brodin L, Low P, Shupliakov O (2000) Sequential steps in clathrinmediated synaptic vesicle endocytosis. Curr Opin Neurobiol 10:312-320.

Chow I, Poo M-M (1985) Release of acetylcholine from embryonic neurons upon contact with muscle cells. J Neurosci 4:1076-1082.

Clements JD, Silver RA (2000) Unveiling synaptic plasticity: a new graphical and analytical approach. Trends Neurosci 23:105-112.

Covault J, Sanes JR (1986) Distribution of N-CAM in synaptic and extrasynaptic portions of developing and adult skeletal muscle. J Cell Biol 102:716-730.

Cremer H, Lange R, Christoph A, Plomann M, Vopper G, Roes J, Brown R, Baldwin S, Kraemer P, Scheff S, Barthels D, Rajewsky K (1994) Inactivation of the N-CAM gene in mice results in size reduction of the olfactory bulb and deficits in spatial learning. Nature 367:455-459.

Crossin KL, Krushel L (2000) Cellular signaling by neural cell adhesion molecules of the immunoglobulin superfamily. Dev Dyn 218:260-279.

Cunningham BA, Hemperly JJ, Murray BA, Prediger EA, Brackenberry R, Edelman GM (1987) Neural cell adhesion molecules: structure, immunoglobulin-like domains, cell surface modulation and alternative splicing. Science 236:799-806.

Dahm LM, Landmesser LT (1991) The regulation of synaptogenesis during normal development and following activity blockade. J Neurosci 11:238-255.

Dai Z, Peng HB (1996) Dynamics of synaptic vesicles in cultured spinal cord neurons in relationship to synaptogenesis. Mol Cell Neurosci 7:443-452.

Dodge Jr FA, Rahamimoff R (1967) Co-operative action of calcium ions in transmitter release at the neuromuscular junction. J Physiol (Lond) 193:419-432.

Featherstone DE, Davis WS, Dubrueil RR, Broadie K (2001) Drosophila $\alpha$ and $\beta$ spectrin mutants disrupt presynaptic neurotransmitter release. J Neurosci 21:4215-4224.

Garner CC, Zhai RG, Gundelfinger ED, Ziv NE (2002) Moelcular mechanisms of CNS synaptogenesis. Trends Neurosci 25:243-250.

Honda I, Kamiya H, Yawo H (2000) Re-evaluation of phorbol esterinduced potentiation of transmitter release from mossy fiber terminals of the mouse hippocampus. J Physiol (Lond) 529:763-776.

Hori T, Taki Y, Takahashi T (1999) Presynaptic mechanism for phorbol ester induced synaptic potentiation. J Neurosci 19:7262-7267.

Kamm KE, Stull JT (2001) Dedicated myosin light chain kinases with diverse cellular functions. J Biol Chem 276:4527-4530.

Koh Y, Ruiz-Canada C, Gorczyca M, Budnik V (2002) The Ras1-Mitogenactivated protein kinase signal transduction pathway regulates synaptic plasticity through fasciclin II mediated cell adhesion. J Neurosci 22:2496-2504.

Kolkova K, Novitskaya V, Pederson N, Berezin V, Bock E (2001) Neural cell adhesion molecule-stimulated neurite outgrowth depends on activation of protein kinase $\mathrm{C}$ and the ras-mitogen-activated protein kinase pathway. J Neurosci 20:2238-2246.

Liu WS, Heckman CA (1998) The sevenfold way of PKC regulation. Cell. Signal 10:529-542.

Lupa MT, Hall ZW (1989) Progressive restriction of synaptic vesicle protein 
to the nerve terminal during development of the neuromuscular junction. J Neurosci 9:3937-3945.

Lyons GE, Moore R, Yahara O, Buckingham ME, Walsh FS (1992) Expression of NCAMisoforms during myogenesis in the mouse embryo. Dev Dynamics 194:94-104.

Majewski H, Iannazzo L (1998) Protein Kinase C: a physiological mediator of enhanced transmitter output. Progress in Neurobiol 55:463-475.

Minami N, Berglund K, Skaba T, Kohmoto H, Tachibana M (1998) Potentiation of transmitter release by protein kinase $\mathrm{C}$ in goldfish retinal bipolar cells. J Physiol 512:219-225.

Mochida S, Kobayashi H, Matsuda Y, Yuda Y, Muramoto K, Nonomura Y (1994) Myosin II is involved in transmitter release at synapses formed between rat sympathetic neurons in culture. Neuron 13:1131-1142.

Moscoso LM, Cremer H, Sanes JR (1998) Organization and reorganization of neuro-muscular junctions in mice lacking neural cell adhesion molecule, tenascin-C, or fibroblast growth factor-5. J Neurosci 18:1465-1477.

Nagy G, Matti U, Nehring RB, Binz T, Rettig J, Neher E, Sorensen JB (2002) Protein kinase C-dependent phosphorylation of synaptotosmeassociated protein of $25 \mathrm{kDa}$ at $\mathrm{Ser}^{187}$ potentiates vesicle recruitment. J Neurosci 22:9278-9286.

Paratcha G, Ledda F, Ibanez CF (2003) The neural cell adhesion molecule NCAM is an alternative signaling receptor for GDNF family ligands. Cell 113:867-879.

Polo-Parada L, Bose CM, Landmesser LT (2001) Alterations in transmission, synaptic vesicle dynamics, and the organization of transmitter release machinery at N-CAM deficient neuromuscular junctions. Neuron 32:815-828.

Rafuse V, Landmesser LT (1996) Contractile activity regulates isoform expression and polysialylation of NCAM in cultured myotubes: involvement of $\mathrm{Ca}^{2+}$ and protein kinase C. J Cell Biol 132:969-983.

Rafuse VF, Polo-Parada L, Landmesser LT (2000) Structural and functional alterations of neuromuscular junctions in NCAM-deficient mice. J Neurosci 20:6529-6539.

Rhee JS, Betz A, Pyott S, Reim K, Varoqueaux F, Augustin I, Hesse D, Sudhof TC, Takaashi M, Rosenmund C, Brose N (2002) $\beta$ phorbol ester- and diacylglycerol-induced augmentation of transmitter release is mediated by Munc13s and not by PKCs. Cell 108:121-133.

Ryan TA (1999) Inhibitors of myosin light chain kinase block synaptic vesicle pool mobilization during action potential firing. J Neurosci 19:1317-1323.

Safell JL, Williams EJ, Mason IJ, Walsh FS, Doherty P (1997) Expression of a dominant negative FGF receptor inhibits axonal growth and FGF receptor phosphorylation stimulated by CAMs. Neuron 18:231-242.

Saitoh N, Hori T, Takahashi T (2001) Activation of the epsilon isoform of protein kinase $\mathrm{C}$ in the mammalian nerve terminal. Proc Natl Acad Sci USA 24:14017-14021.

Sanes JR, Lichtman JW (1999) Development of the vertebrate neuromuscular junction. Annu Rev Neurosci 22:389-442.
Schmid RS, Graff RD, Schaller MD, Chen S, Schachner M, Hemperly JJ, Maness PF (1999) N-CAM stimulates the Ras-MAPK pathway and CREB phosphorylation in neuronal cells. J Neurobiol 38:542-558.

Schoch S, Castillo PE, Jo T, Mukherjee K, Geppert M, Wang Y, Schmitz F, Malenka RC, Sudhof TC (2002) RIM $\alpha$ forms a protein scaffold for regulating neurotransmitter release at the active zone. Nature 415:321-326.

Searl TJ, Silinsky EM (1998) Increases in acetylcholine release produced by phorbol esters are not mediated by protein kinase $\mathrm{C}$ at motor nerve endings. J Pharmacol Exp Ther 285:247-251.

Shen H, Watanabe M, Tomasiewicz H, Glass JD (2000) Genetic deletions of NCAM and PSA impair circadian function in the mouse. Physiol Behav 73:185-193.

Smith C (1999) A persistant activity-dependent facilitation in chromaffin cells is caused by $\mathrm{Ca}^{2+}$ activation of protein kinase C. J Neurosci 19:589-598.

Stevens CF, Sullivan JM (1998) Regulation of the readily releasable vesicle pool by protein kinase C. Neuron 21:885-893.

Sun Y, Poo M-M (1987) Evoked release of acetylcholine from the growing embryonic neurons. Proc Natl Acad Sci USA 84:2540-2544.

Sytnyk V, Leshchyn'ka I, Dekking M, Dityateva G, Dityatev A, Schachner M (2002) Neural cell adhesion molecule promotes accumulation of TGN organelles at sites of neuron-to-neuron contacts. J Cell Biol 159:649-661.

Thomas U, Kim E, Kuhlendahl S, Koh YH, Gundelfinger ED, Sheng M, Garner CC, Budnik V (1997) Synaptic clustering of the cell adhesion molecule fasciclin II by discs-large and its role in the regulation of presynaptic structure. Neuron 19:787-799.

Tokimasa T (1995) Effects of myosin light chain kinase inhibitors on delayed rectifyer potassium current in bullfrog sympathetic neurons. Neurosci Lett 197:75-77.

Tomasiewicz H, Ono K, Yee D, Thompson C, Goridis C, Rutishauser U, Magnuson T (1993) Genetic deletion of a neural cell adhesion molecule variant (N-CAM-180) produces distinct defects in the central nervous system. Neuron 11:1163-1174.

Urbano FJ, Rosata-Siri MD, Uchitel OD (2002) Calcium channels involved in neurotransmitter release at adult, neonatal and P/Q-type deficient neuromuscular junctions. Mol Membr Biol 19:293-300.

Whittaker VP (1984) The synaptosome. In: Handbook of neurochemistry, Vol 7.4, Ed 2 (Lajtha A, ed), pp 1-39. New York: Plenum.

Yao W-D, Rusch J, Poo M-M, Wu C-F (2000) Spontaneous acetylcholine secretion from developing growth cones of Drosophila central neurons in culture: effects of camp-pathway mutations. J Neurosci 20:2626-2637.

Yu W, Niwa T, Fukusawa T, Hidaka H, Senda T, Sasaki Y, Niki I (2000) Synergism of protein kinase A, protein kinase $\mathrm{C}$, and myosin light chain kinase in the secretory cascade of the parcreatic B-cell. Diabetes 49:945-952.

Zakharenko S, Chang S, O’Donoghue M, Popov SV (1999) Neurotransmitter secretion along growing nerve processes: comparison with synaptic vesicle exocytosis. J Cell Biol 144:507-518. 